A\&A 650, A112 (2021)

https://doi.org/10.1051/0004-6361/202039726

(c) E. Zari et. al 2021

\title{
Mapping luminous hot stars in the Galaxy ${ }^{\star}$
}

\author{
E. Zari ${ }^{1}$, H.-W. Rix ${ }^{1}$, N. Frankel ${ }^{1}$, M. Xiang ${ }^{1}$, E. Poggio ${ }^{2,3}$, R. Drimmel $^{2}$, and A. Tkachenko ${ }^{4}$ \\ 1 Max-Planck-Institut für Astronomie, Königstuhl 17, 69117 Heidelberg, Germany \\ e-mail: zari@mpia-hd.mpg.de \\ 2 Osservatorio Astrofisico di Torino, Istituto Nazionale di Astrofisica (INAF), 10025 Pino Torinese, Italy \\ 3 Université Côte d'Azur, Observatoire de la Côte d'Azur, CNRS, Laboratoire Lagrange, Nice, France \\ ${ }^{4}$ Institute of Astronomy, KU Leuven, Celestijnenlaan 200D, 3001 Leuven, Belgium \\ Received 20 October 2020 / Accepted 16 February 2021
}

\begin{abstract}
Luminous hot stars $\left(M_{K_{s}} \lesssim 0 \mathrm{mag}\right.$ and $\left.T_{\text {eff }} \gtrsim 8000 \mathrm{~K}\right)$ dominate the stellar energy input to the interstellar medium throughout cosmological time, are used as laboratories to test theories of stellar evolution and multiplicity, and serve as luminous tracers of star formation in the Milky Way and other galaxies. Massive stars occupy well-defined loci in colour-colour and colour-magnitude spaces, enabling selection based on the combination of Gaia EDR3 astrometry and photometry and 2MASS photometry, even in the presence of substantive dust extinction. In this paper we devise an all-sky sample of such luminous OBA-type stars, which was designed to be complete rather than very pure, providing targets for spectroscopic follow-up with the SDSS-V survey. To estimate the purity and completeness of our catalogue, we derive stellar parameters for the stars in common with LAMOST DR6 and we compare the sample to other $\mathrm{O}$ and B-type star catalogues. We estimate 'astro-kinematic' distances by combining parallaxes and proper motions with a model for the expected velocity and density distribution of young stars; we show that this adds useful constraints on the distances and therefore luminosities of the stars. With these distances we map the spatial distribution of a more stringently selected subsample across the Galactic disc, and find it to be highly structured, with distinct over- and under-densities. The most evident over-densities can be associated with the presumed spiral arms of the Milky Way, in particular the Sagittarius-Carina and Scutum-Centaurus arms. Yet, the spatial picture of the Milky Way's young disc structure emerging in this study is complex, and suggests that most young stars in our Galaxy $\left(t_{\text {age }}<t_{\text {dyn }}\right)$ are not neatly organised into distinct spiral arms. The combination of the comprehensive spectroscopy to come from SDSS-V (yielding velocities, ages, etc.) with future Gaia data releases will be crucial in order to reveal the dynamical nature of the spiral arms themselves.
\end{abstract}

Key words. stars: early-type - Galaxy: structure - Galaxy: disk

\section{Introduction}

Luminous and hot stars are massive, and hence rare and short lived. However, they play decisive roles across different fields of astrophysics. They dominate the interaction between stars and the interstellar gas and dust (interstellar medium; ISM) in their host galaxies by heating or ionising those components; those stars also interact with the ISM through powerful stellar winds and eventually supernova explosions (Mac Low \& Klessen 2004; Hopkins et al. 2014). Luminous and hot stars must indeed have played an important role in galaxy evolution throughout cosmic time, via their intense winds, ultraviolet radiation fields, chemical processing, and explosions (Haiman \& Loeb 1997; Douglas et al. 2010; Bouwens et al. 2011).

Luminous and hot stars are inevitably young, and therefore they can serve as tracers of recent massive star formation. Although they make up an insignificant fraction of the overall stellar mass, they contribute a major portion of the light of the disc and can therefore probe the spiral structure and young disc kinematics of our Galaxy and others (Xu et al. 2018; Chen et al. 2019; Dobbs \& Baba 2014; Kendall et al. 2011, 2015).

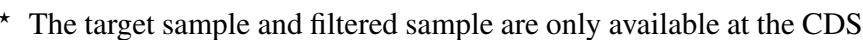
via anonymous ftp to cdsarc. u-strasbg. fr (130.79.128.5) or via http://cdsarc.u-strasbg.fr/viz-bin/cat/J/A+A/650/A112
}

Also, these objects are - directly or through their remnants - decisive drivers of the chemical evolution for many elements in the periodic table and serve as crucial laboratories for stellar evolution in this arguably least well-understood regime of stellar physics.

Massive stars are born predominantly as members of binary and multiple systems (Sana et al. 2012, 2014; Kobulnicky et al. 2014; Moe \& Di Stefano 2017). As a consequence, most of them are expected to undergo strong binary interaction, which drastically alters their evolution (Podsiadlowski et al. 1992; Van Bever \& Vanbeveren 2000; O'Shaughnessy et al. 2008; de Mink et al. 2013; Langer et al. 2020).

Finally, massive (binary) stars are the only channel to yield binaries that involve black holes and neutron stars in the disc of the Milky Way. Therefore, understanding massive star binaries, including as they evolve away from their zero-age main sequence, is indispensable for understanding the distribution of gravitational wave events. For all of the above reasons, a multiepoch, spectroscopic census of luminous and hot stars across the Galaxy is needed, providing spatial and dynamic information together with estimates for masses, ages, metallicity, multiplicity, and other spectroscopic information. The SDSS-V survey (Kollmeier et al. 2017, and in prep.; Bowen \& Vaughan 1973; Gunn et al. 2006; Smee et al. 2013; Wilson et al. 2019) will provide such a comprehensive, multi-epoch spectroscopic program on hot and massive stars, but this program must be based on a 
clear and quantitative selection function to enable rigorous subsequent population studies.

There is no universal precise 'definition' across different subcommunities within astrophysics of when a star is 'luminous', 'hot', and 'massive'. 'Hot' may either mean that its $T_{\text {eff }}$ is sufficiently high that the spectrum is dominated by $\mathrm{H}$ and $\mathrm{He}$ lines, rather than metal lines (i.e. OBA stars); or it may mean that $T_{\text {eff }}$ is sufficiently high that the star produces significant amounts of ionising radiation ( $\mathrm{O}$ and early $\mathrm{B}$ stars). 'Massive' may either mean, massive enough to 'go supernova' or to have a convective core.

For the current context we choose an operative definition of luminous and hot as $T_{\text {eff }} \gtrsim 8000 \mathrm{~K}$ and $M_{K} \lesssim 0 \mathrm{mag}$, or - loosely speaking - OBA stars. This is the set of stars that addresses the science sketched out above. Some aspects, such as ISM ionisation, are only affected by $\mathrm{O}$ and early B stars; but it is important to remember that even the most massive stars do not produce ionising radiation during all of their evolution. Therefore we aim in our sample definition for a sample that is hot enough to eliminate all the luminous red giant branch (RGB) and asymptotic giant branch (AGB) stars, but include most evolved phases of massive stars. Stars with $T_{\text {eff }} \geq 8000 \mathrm{~K}$ can be photometrically pre-selected across the entire sky; yet efficient discrimination between $10000 \mathrm{~K}$ and $20000 \mathrm{~K}$ by means of photometry only is almost impossible in the presence of dust.

In light of the science goals above, in this paper we present the selection of the target sample of massive stars of the SDSS$\mathrm{V}$ survey. We describe an approach to use kinematics to improve distance estimates. Finally we use a 'clean' subset of the target sample to study the structure of the Milky Way disc as traced by young, massive stars.

The selection of our target sample is based on the combination of Gaia EDR3 (Gaia Collaboration 2016, 2021a) astrometry and photometry and 2MASS (Skrutskie et al. 2006) photometry. Similar samples of stars in the upper main sequence (UMS) were already selected by Poggio et al. (2018) and Romero-Gómez et al. (2019) to characterise the structure and the kinematic properties of the Galactic warp. Poggio et al. (2018) used a combination of Gaia DR2 and 2MASS colours to select stars of spectral type earlier than B3, and obtained a sample of 599494 stars. Romero-Gómez et al. (2019) used only Gaia DR2 photometry to select stars brighter than $M_{G, 0}=2 \mathrm{mag}$ (thus likely including also A-type stars), and obtained a sample of 1860651 stars. Our sample definition (described in Sect. 2) lies somewhat in the middle between those by Poggio et al. (2018) and RomeroGómez et al. (2019). In Sect. 3 we describe the method that we use to estimate distances. In Sect. 4 we clean the target sample of sources with spurious astrometry and we filter out older contaminants. In Sect. 5 we use this filtered sample to study the 3D space distribution of the sources and we compare the distribution of OBA stars with other tracers of massive star formation and spiral arm structure. In Sect. 6 we discuss our findings in the context of the structure and nature of the spiral arms of the Milky Way. Finally, we summarise our results and draw our conclusions in Sect. 7.

\section{Target sample}

In this section we present the criteria that we applied to select the target sample for the SDSS-V spectroscopic survey, and we describe its characteristics in terms of sky distribution, magnitude and luminosity distribution, variability, purity, and completeness.

\subsection{Astro-photometric selection}

To select our sample, we use Gaia EDR3 photometry (Riello et al. 2021) and astrometry (Lindegren et al. 2021a) combined with 2MASS photometry. The cross-match between Gaia and 2MASS is not yet provided in the Gaia archive (Marrese et al., in prep.) ${ }^{1}$. For this reason we first performed a cross-match with Gaia DR2, followed by a cross-match with 2MASS. The ADQL query for this cross-match is provided in Appendix A.

We restrict our query to the sources with $G<16 \mathrm{mag}$. The motivation behind this condition is twofold. On the one hand, it is a SDSS-V technical requirement: at $G=16$ mag we can obtain a $S / N=75$ with 15 min exposures with the BOSS spectrograph (Smee et al. 2013). On the other hand, for $G>16$ mag the Gaia EDR3 parallax precision rapidly deteriorates (see e.g. Table 3 in Gaia Collaboration 2021a), thus (a) estimating absolute magnitudes (which are crucial to our selection) becomes nontrivial and (b) inferring distances strongly depends on the choice of the prior (see Appendix B and Sect. 3).

We define a proxy for the absolute magnitude of a star in the $K_{s}$ band, $\tilde{M}_{K_{s}}$, and we require $\tilde{M}_{K_{s}}<0$. This translates to the following parallax condition:

$\varpi<10^{\left(10-K_{s}-0.0\right) / 5}$,

and aims at selecting a reasonably sized sample of bright stars. The value $M_{K_{s}}=0$ mag corresponds roughly to a B7V-type star (Pecaut \& Mamajek 2013). To include also later B-type stars we should require $\tilde{M}_{K_{s}}<1 \mathrm{mag}$, as $M_{K_{s}}=1 \mathrm{mag}$ roughly corresponds to the absolute magnitude of a A0V-type star. We chose however $\tilde{M}_{K_{s}}=0$ mag as a good compromise between completeness and contamination (see Sect. 2.3).

We stress that for the survey target list we do not require any condition on the photometric quality of 2MASS, or on the photometric errors, the parallax accuracy, or the goodness of fit of the astrometric solution. This is motivated by the fact that we are aiming to obtain a known, well-defined selection function: not only do measurement errors not describe intrinsic physical properties of the sources, and therefore should not enter the selection criteria, but they are also difficult to model.

At this point our sample, shown in the $\left(J-K_{s}\right)_{0}$ versus $G_{\mathrm{BP}}-$ $G_{\mathrm{RP}}$ colour-magnitude diagram in Fig. 2, consists of luminous stars, which could either be OBA, RGB, or AGB stars. OBA stars fall in the region highlighted by the orange ellipse. The stars in the horizontal stripes are stars with large 2MASS photometric errors or 2MASS photometric quality flags (ph_flag) different from "AAA".

To select OBA stars we use simple photometric cuts, roughly following the procedure outlined in Poggio et al. (2018, see in particular their Fig. 1). The first step of our selection consists in selecting sources with:

$\left(J-K_{s}\right)_{0}<0.1 \mathrm{mag}$ and $\left(J-K_{s}\right)_{0}>-0.3 \mathrm{mag}$.

The first condition excludes most of the red sources, and the second condition removes sources with unnaturally blue colours. We obtained the $\left(J-K_{s}\right)_{0}$ colour by applying the following Equation:

$\left(J-K_{s}\right)_{0}=\left(J-K_{s}\right)-0.25\left(G-K_{s}\right)$,

which we derived by noticing that, for the spectroscopically confirmed O- and B-type stars selected by Liu et al. (2019), the

1 https://archives.esac.esa.int/gaia 

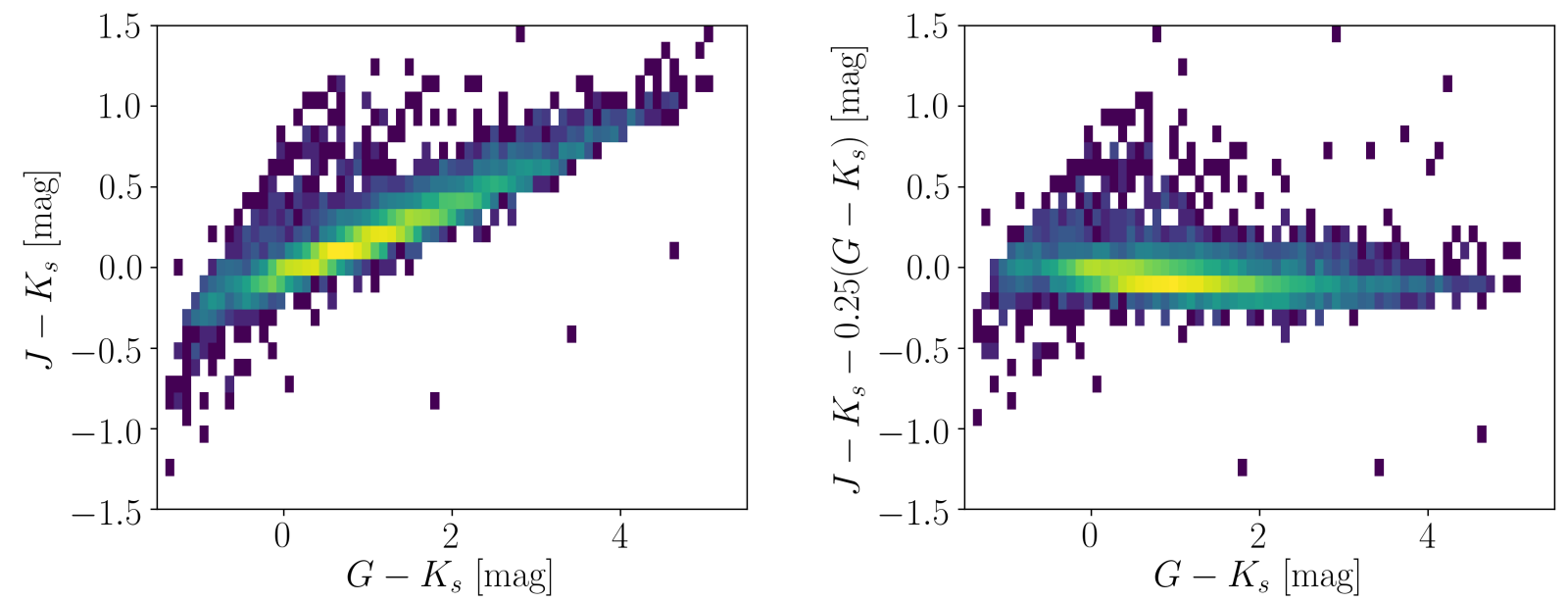

Fig. 1. Two-dimensional histogram of $J-K_{s}$ vs. $G-K_{s}(l e f t),\left(J-K_{s}\right)_{0}$, and $G-K_{s}(r i g h t)$ for the stars in the LAMOST sample by Liu et al. (2019); see main text for more details on the sample.

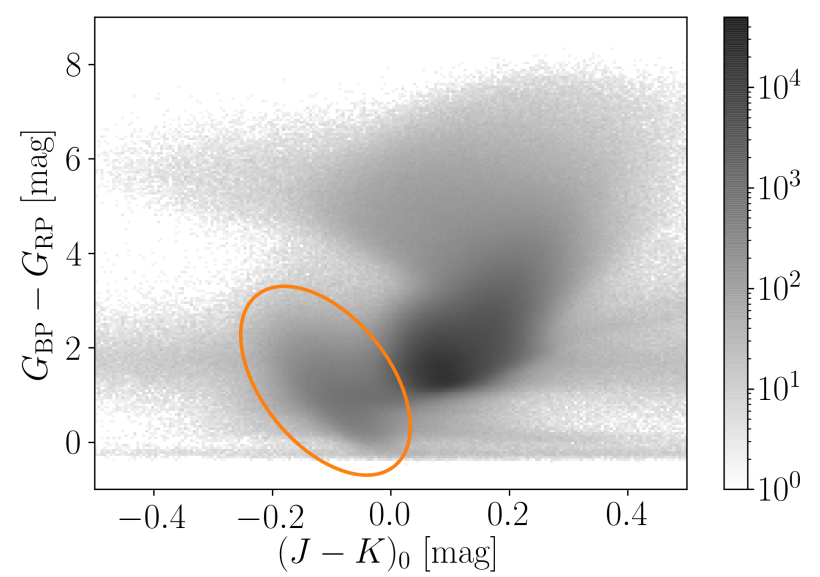

Fig. 2. $\left(J-K_{s}\right)_{0}$ vs. $G_{\mathrm{BP}}-G_{\mathrm{RP}}$ colour-magnitude diagram. OBA stars roughly fall in the region highlighted by the orange ellipse. This representation shows that hot stars stand out qualitatively in colour-colour space.

$\left(J-K_{s}\right)$ and $\left(G-K_{s}\right)$ colours are linearly correlated (see Fig. 1, left), and by assuming that the difference between the $J$ and $K_{s}$ magnitudes for such stars is close to zero (see e.g. Pecaut \& Mamajek 2013).

Figure 3 shows the distribution of sources in the $G-K_{s}$ versus $J-H$ colour-colour diagram. O- and B-type stars lie on a sequence in the $J-H$ versus $G-K_{s}$ colour-colour diagram as a consequence of interstellar reddening and are clearly separated from redder turn-off stars and giants. We therefore select stars located in the region defined by the equations:

$J-H<0.15\left(G-K_{S}\right)+0.05$

and

$J-H>0.15\left(G-K_{S}\right)-0.15$,

(solid grey lines in Fig. 3). Finally, we select stars in the $G$ versus $G-K_{s}$ colour-magnitude diagram, where giants still contaminating our sample can easily be separated from OBA stars (see Fig. 4). In particular we select stars satisfying:

$G>2\left(G-K_{s}\right)+3$,

(solid orange line in Fig. 4). The catalogue consists of 988202 entries.

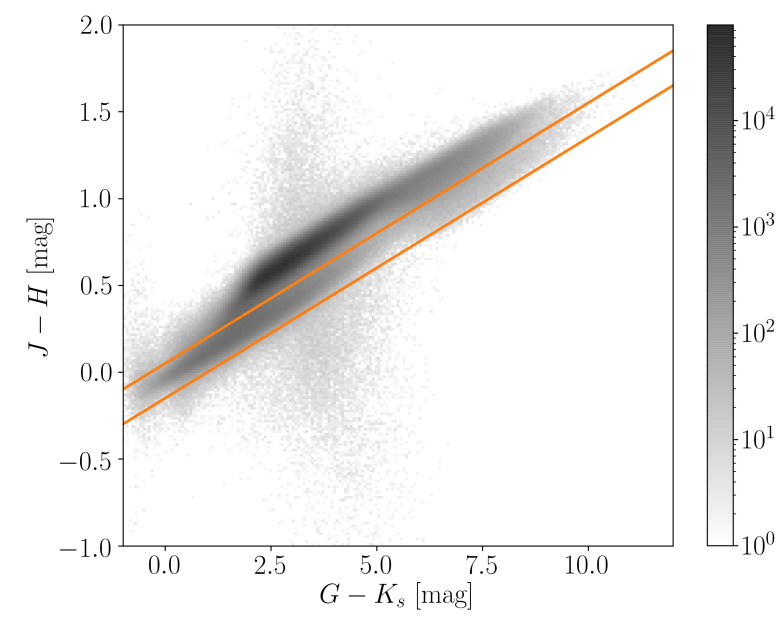

Fig. 3. $J-H$ vs. $G-K_{s}$ colour-colour diagram of the sources with $\left(J-K_{s}\right)_{0}<0.1 \mathrm{mag}$ and $\left(J-K_{s}\right)_{0}>-0.3 \mathrm{mag}$. The solid lines delineate the criteria for selecting OBA stars and are defined in the text.

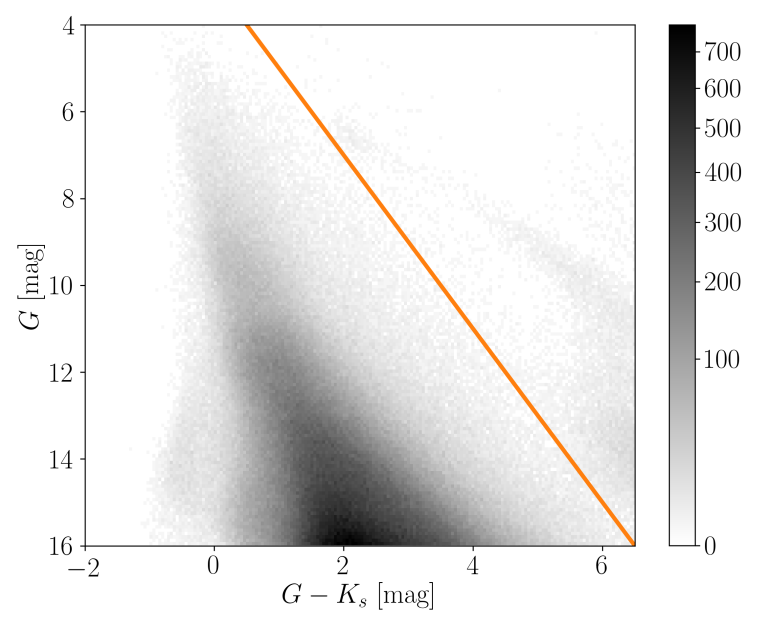

Fig. 4. $G-K_{s}$ vs. $G$ colour-magnitude diagram of the sources selected after applying the criteria in the $\left(J-K_{s}\right)_{0}$ vs. $G-K_{s}$ and $G-K_{s}$ vs. $J-H$ colour-magnitude diagrams (see text). The solid orange line shows the equation $G=2\left(G-K_{s}\right)+3$, and is designed to exclude the few cool giants that may still contaminate the sample. 


\subsection{Characteristics of the target sample}

Figure 5 shows the sky distribution of the sample selected in the previous sections in Galactic coordinates $(l, b)$.

The stars in our catalogue are distributed in our Galaxy (mainly on the Galactic plane) and in the Large (LMC, $l, b \sim$ $280^{\circ},-34^{\circ}$ ) and the Small (SMC, $l, b \sim 303^{\circ},-45^{\circ}$ ) Magellanic Clouds. We stress that massive stars in the Magellanic clouds are included in the target sample of the SDSS-V survey. However, in the present paper we focus on the Milky Way disc, thus in the following sections we restrict our sample to stars with $|b|<25^{\circ}$. Massive star-forming regions can be recognised as over-densities in the source distribution.

Dust features are visible as 'gaps' in the star distribution. For example, the Aquila rift, the Pipe Nebula, and the Ophiucus clouds can be easily identified towards the Galactic centre $(l, b=0,0 \mathrm{deg})$. By visually inspecting the features of Fig. 5, it is possible to identify old stars contaminating the sample. For instance, at $l, b \sim 309^{\circ}, 15^{\circ}$ the $\omega$ Centaurus globular cluster is visible, while the stars towards the Galactic centre delineate the shape of the bulge. We further study the purity and completeness of our sample in Sect. 2.3.

Figure 6 shows the distribution in apparent (left) and absolute magnitude (right) of the sources. The orange histograms show the distribution of all the sources in our sample. The grey histograms show the distribution of the sources selected in Sect. 3. In the right hand panel, absolute magnitudes are computed using the inversion of parallaxes as the distance (orange histogram) or astro-kinematic distances (grey histogram; see Sect. 3). The grey distribution appears shifted towards larger $M_{G}$ values. This is due to the fact that, for stars with small parallaxes (and large parallax errors), our astro-kinematic distances are shorter than those obtained by inverting the parallax.

Figure 7 (left) shows the variability distribution of the sources in the $G$ band vs. the ratio of the variability in the $G_{\mathrm{BP}}$ and $G_{\mathrm{RP}}$ bands. Following roughly Belokurov et al. (2017), Deason et al. (2017), and Iorio et al. (2018), the variability in a given filter $x$ is defined as:

$$
\mathrm{AMP}_{\mathrm{x}}=\frac{\sqrt{\text { phot_x_n_obs }}}{\text { phot_x_mean_flux_over_error }},
$$

where phot_x_n_obs is the number of observations contributing to the $x$-band photometry, and

$$
\begin{aligned}
& \text { phot_x_mean_flux_over_error }= \\
& \text { phot_x_mean_flux/phot_x_mean_flux_error. }
\end{aligned}
$$

While most of our sources are not variable, some cluster in two regions: RR Lyrae stars have $G$-band variability ranging from 5 to $40 \%$ (Belokurov et al. 2017) and $G_{\mathrm{BP}} / G_{\mathrm{RP}}$ variability around 1.6; eclipsing binaries have $G$ variability up to $30 \%$ and roughly equal $G_{\mathrm{BP}}$ and $G_{\mathrm{RP}}$ variability. Figure 7 (right) shows the same as Fig. 7 (left) but for the sources selected in Sect. 3 (see below), that have astro-kinematic distances compatible with those estimated by Bailer-Jones et al. (2018). This selection removes RR Lyrae stars, while retaining eclipsing binaries: this is expected as RR Lyrae stars do not follow the same kinematics as the OBA stars that we are interested in.

\subsection{Completeness and purity}

To study the purity level of our sample we cross-matched it with LAMOST DR6 ${ }^{2}$, and find 36617 sources in common. We

\footnotetext{
2 http://dr6.lamost.org/
}

derived effective temperatures $\left(T_{\text {eff }}\right)$ and surface gravity $(\log g)$ from the LAMOST spectra by fitting ab initio model spectra generated with the 1D-LTE ATLAS12 model atmosphere (Xiang et al., in prep.). Figure 8 shows the distribution of sources in the $\log T_{\text {eff }}$ vs. $M_{K_{s}}$ plane. Around $45 \%$ of the sources are hotter than $9700 \mathrm{~K}$ (orange solid line in Fig. 8, left, corresponding to the temperature of a A0V-type star) and around $20 \%$ are hotter than $14000 \mathrm{~K}$ (roughly the temperature of a B7V-type star). We assume that we can extrapolate the same numbers to the entire sample. The PARSEC isochrones $\left(A_{V}=0 \mathrm{mag}\right.$ and solar metallicity) in Fig. 8 (Bressan et al. 2012; Tang et al. 2014) show that our main sources of contamination are evolved massive or intermediate mass stars (e.g. yellow and blue super-giants) and that the sample is substantially free from RGB and AGB stars, which are colder and more luminous than the stars in our sample. In the left-hand panel, the isochrones are colour-coded by their log age (from $1 \mathrm{Myr}$ to $500 \mathrm{Myr}$ ), in the right-hand panel they are colour-coded by mass.

To estimate the completeness of the sample we crossmatched with the O and B-type star catalogue by Liu et al. (2019) and the O-type star catalogue by Sota et al. (2014) and Maíz Apellániz et al. (2016). The Liu et al. (2019) sample consists of 16032 stars that reduce to 15206 after cross-matching with Gaia EDR3 and 2MASS and further checking for duplicated sources ( $1^{\prime \prime}$ cross-match radius), and to 9083 stars by applying Eq. (1). The cross-match between the Liu et al. (2019) catalogue and ours gives 8212 sources. The stars that are not included in the cross-match are those whose infrared colours are not consistent with our selection. The sample of Sota et al. (2014) and Maíz Apellániz et al. (2016, the Galactic O-star spectroscopic survey, GOSSS) consists of 590 stars that reduce to 580 after crossmatching with Gaia EDR3 and 2MASS. Our catalogue contains $503(\sim 86 \%)$ of the GOSSS stars. As with the LAMOST sample, the stars missing from our selection do not follow some of our photometric selection criteria. For example, many of the missing GOSSS sources $\left(49 \%, 54\right.$ stars) have $J-H>0.15\left(G-K_{s}\right)+0.05$ (see Eq. (4)), that is 2MASS-Gaia EDR3 colours consistent with being giants.

\section{Distance estimates}

To study the 3D space distribution of our sources, precise distance estimations are needed. We estimate distances using a model designed to reproduce the properties of our data-set in terms of spatial and luminosity distribution, and the additional information that stars belonging to our sample should follow Galactic rotation, with a small, typical velocity dispersion. By making such an assumption we neglect non-circular streaming motions. We can then predict the true proper motions $\left(\mu_{l}^{\prime}, \mu_{b}^{\prime}\right)$ of the stars in our sample and compare them with the observed ones, thus adding a further constraint to the distance estimation and deriving 'astro-kinematic' distances. The probability density function $(p d f)$ for a star in our sample to be at a certain distance $d_{\text {kin }}$ is given by:

$p\left(d \mid \boldsymbol{o}, m_{K_{s}}, \Theta_{\mathrm{KM}}, \Theta_{\mathrm{SM}}, \Theta_{\mathrm{CMD}}\right) \propto$
$p\left(\boldsymbol{o} \mid \Theta_{\mathrm{KM}}\right) p\left(d, m_{K_{s}} \mid l, b, \Theta_{\mathrm{SM}}, \Theta_{\mathrm{CMD}}\right)$,

where $\boldsymbol{o}=\left(\varpi-\varpi_{0}, \mu_{l^{*}}, \mu_{b}\right)$ is the array of the astrometric observables, i.e. parallax $\varpi$ (with $\varpi_{0}$ the parallax zero-point) and proper motions components in $l$ and $b, \mu_{l} *$, and $\mu_{b} ; m_{K_{s}}$ is the apparent magnitude in the $K_{s}$ band; $\Theta_{\mathrm{KM}}$ represents our kinematic model; $\Theta_{\mathrm{SM}}$ represents our model for the distribution of stars in the Galaxy; and $\Theta_{\mathrm{CMD}}$ accounts for the observational 


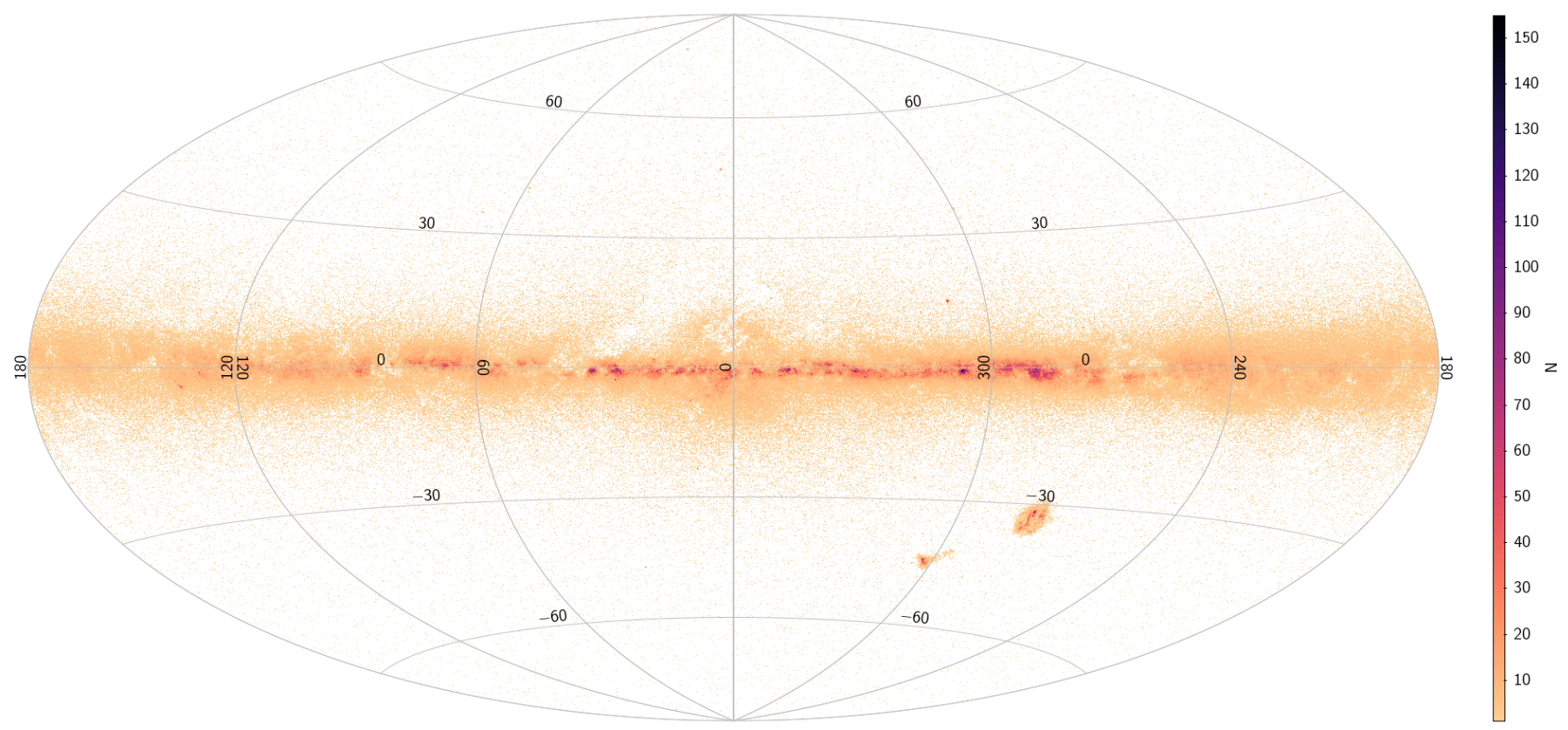

Fig. 5. On-sky density distribution of the candidate OBA stars selected in Sect. 2, in Galactic coordinates. Most of the sources are located in the Galactic plane $\left(b=0^{\circ}\right)$ and in the Large and Small Magellanic clouds.
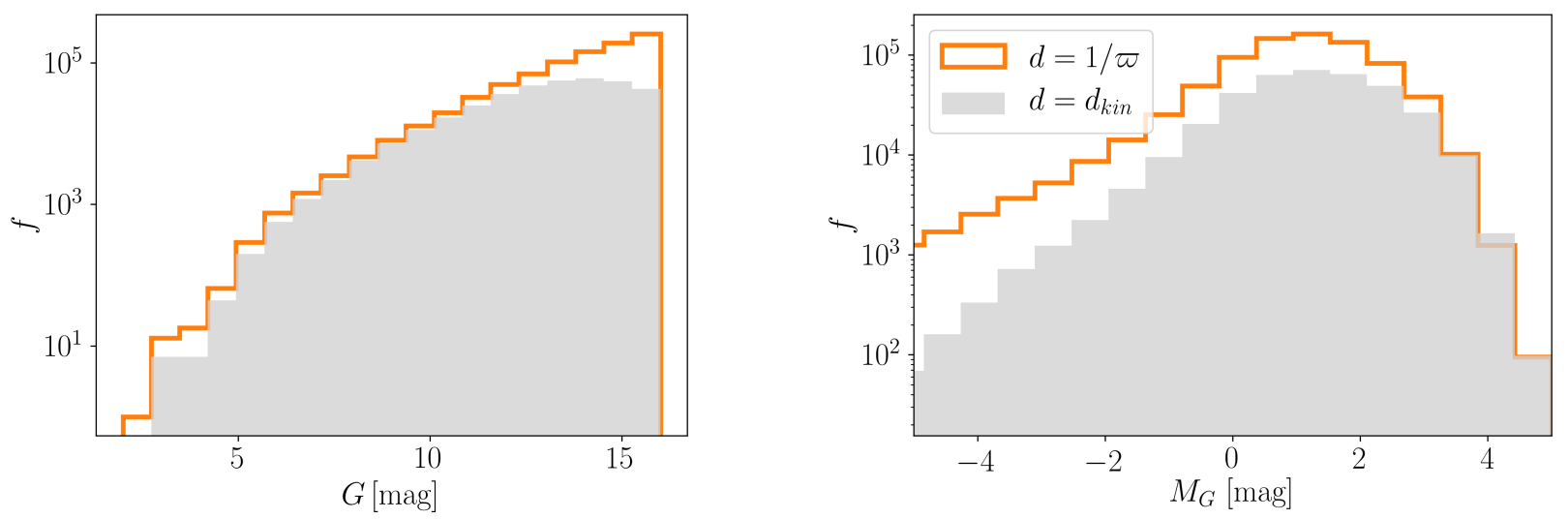

Fig. 6. Left: $G$ magnitude distribution of all the OBA stars selected in Sect. 2.1 (orange histogram) and of the sources selected in Sect. 3 (grey histogram), with astro-kinematic distances comparable with those estimated by Bailer-Jones et al. (2018). Right: absolute magnitude distribution of all the OBA stars selected in Sect. 2.1, computed using their parallax (orange histogram), and of the sources selected in Sect. 3, computed using their astro-kinematic distances (grey histogram).
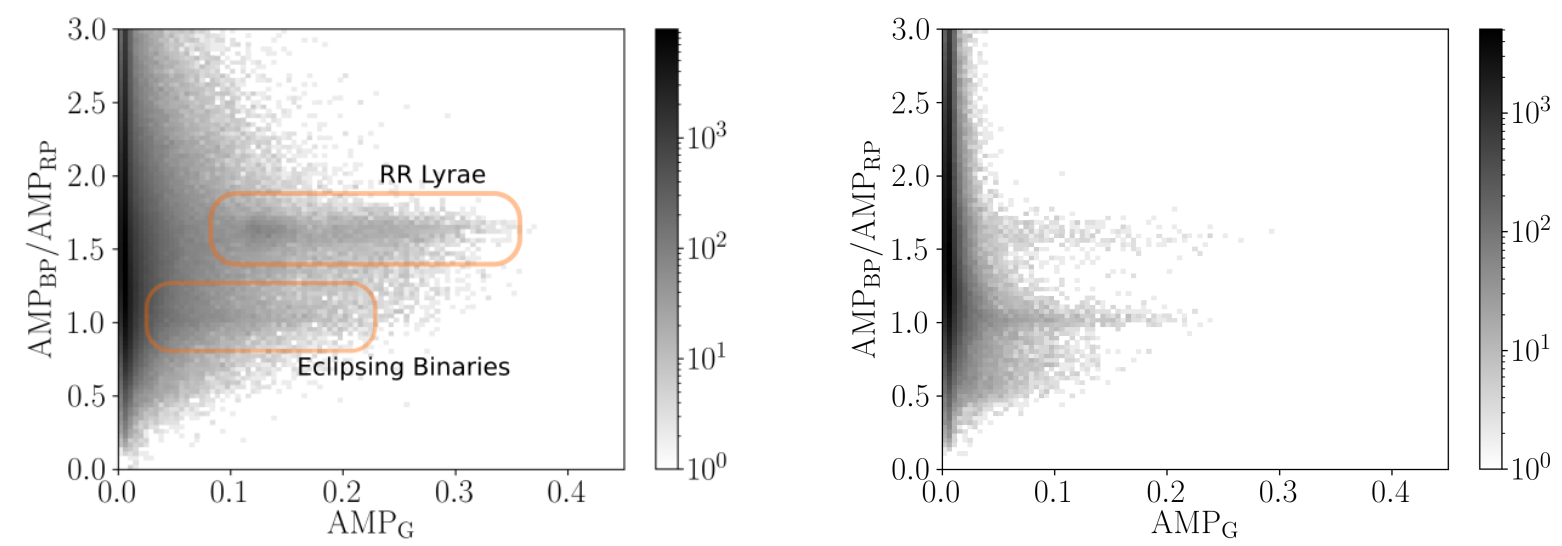

Fig. 7. $G$-band variability vs. $G_{\mathrm{RP}} / G_{\mathrm{BP}}$ variability of all the sources selected in Sect. 2.1 (left) and of the sources selected in Sect. 4 (right). Eclipsing binaries have the same level variability in $G_{\mathrm{BP}}$ and $G_{\mathrm{RP}}$ and $G$-variability lower than 0.3 . RR Lyrae stars have $G_{\mathrm{BP}} / G_{\mathrm{RP}}$ variability $\sim 1.6$ and $G$-band variability lower than 0.4 . 

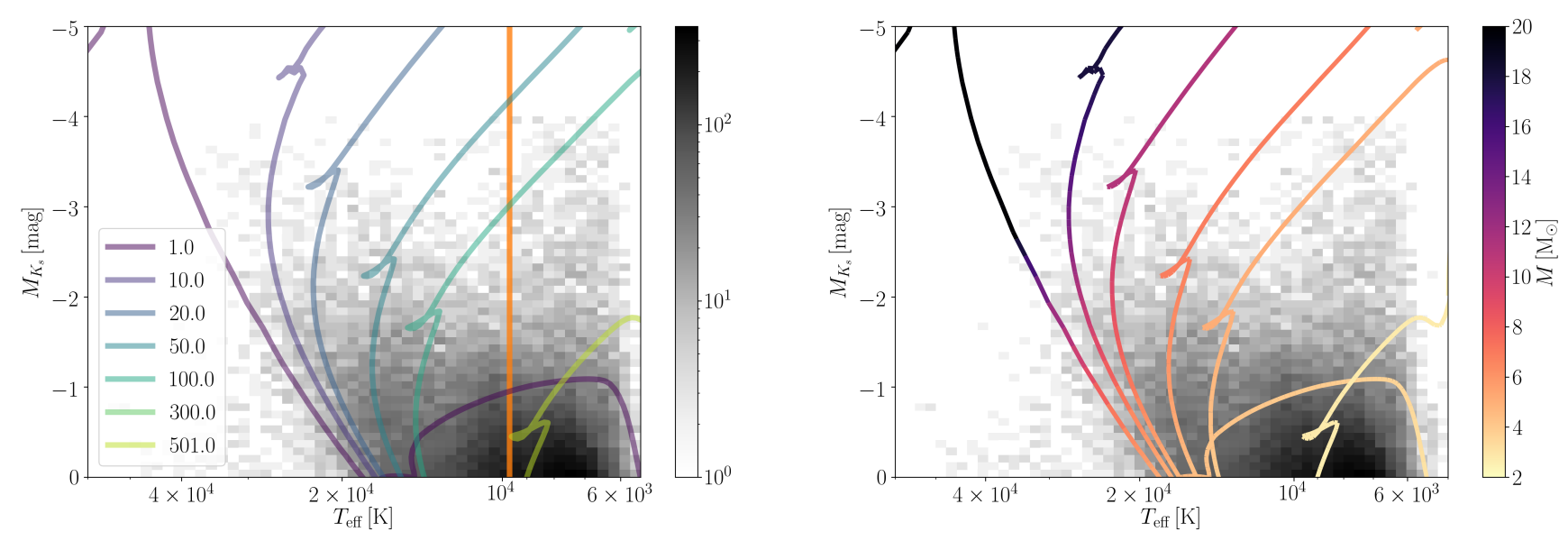

Fig. 8. Left: distribution of the sources with LAMOST DR6 spectra in the $\log T_{\text {eff }}$ vs. $M_{K_{s}}$ (grey). The orange solid line indicates $T_{\text {eff }}=9700 \mathrm{~K}$, which corresponds to the temperature of a A0V-type star (Pecaut \& Mamajek 2013). The isochrones are colour-coded according to their age (in Myr). Right: same as left, with the isochrones colour-coded according to their mass.
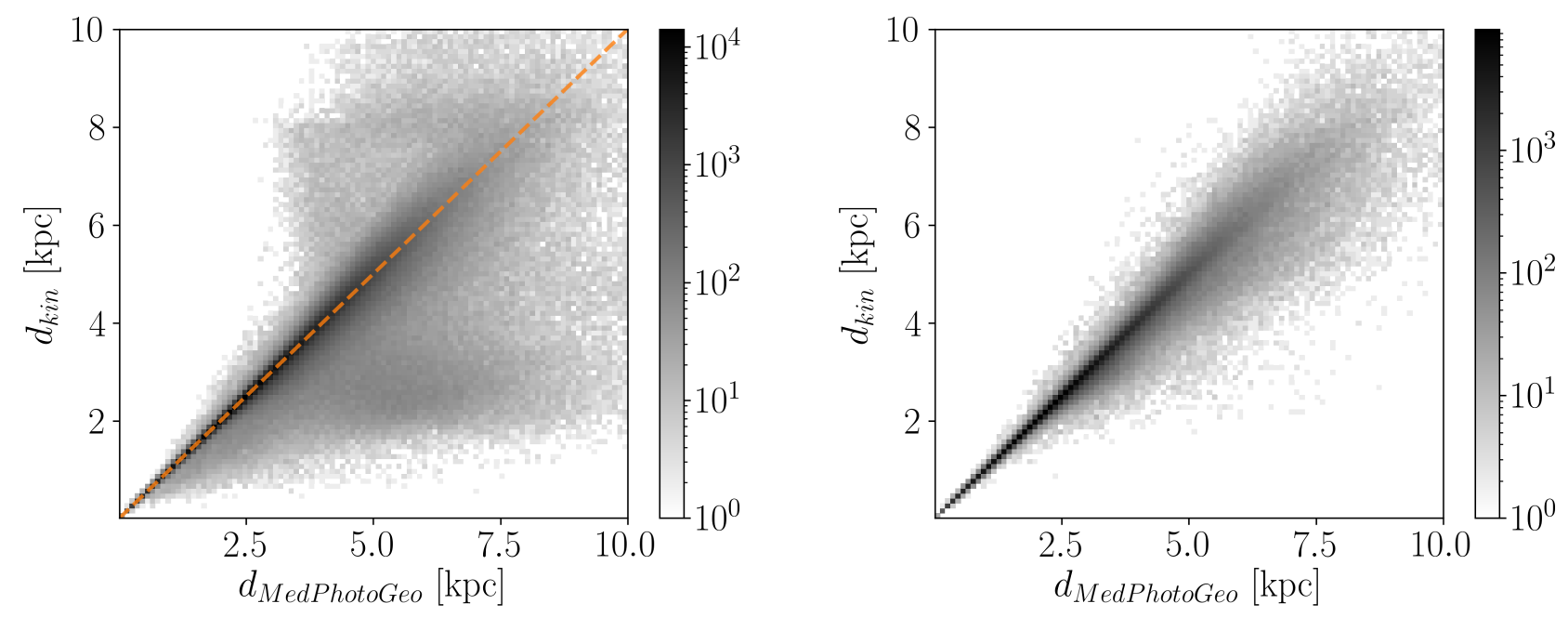

Fig. 9. Comparison between the photo-geometric distances estimated by Bailer-Jones et al. (2020) and our astro-kinematic distances (see Sect. 3), after removing spurious sources (left), and after removing sources with vertical velocity not consistent with our kinematic model and with $M_{K_{s}}<$ 0 mag (right). The orange dashed line has equation $y=x$.

effects due to our selection function on the spatial distribution of our sample.

The method and all the terms in Eq. (8) are described in detail in Appendix B. We adopt the mode of the $p d f$ of Eq. (8) as the astro-kinematic distance estimate $d_{\text {kin }}$, and we use the 16 th and 84th percentiles to estimate distance errors. We also tried using the median of the $p d f$ as a distance estimate, but this did not cause significant differences in the maps presented in Sect. 5. Figure 9 (left) shows the comparison between our astrokinematic distances and the photo-geometric distances estimated by Bailer-Jones et al. (2020). More than $85 \%$ of the sources have astro-kinematic distances consistent within $1 \sigma$ with the photogeometric distances from Bailer-Jones et al. (2020). We comment on the sources with inconsistent distances in Sect. 4.2.

\section{Filtered sample}

In this section we define a subset of the target sample (Sect. 2), which we use to map the structure of the young Milky Way disc. Such a "filtered" sample is obtained by cleaning the target sample for sources with likely spurious astrometric solutions
(Sect. 4.1) and by removing sources with kinematic properties not consistent with the model that we used to estimate astrokinematic distances (Sect. 4.2).

\subsection{Removal of spurious sources}

To remove spurious sources, we use a method developed by Rybizki et al. (2021) based on Gaia Collaboration (2021b). Spurious sources have poor astrometric solutions that can be due to the inconsistent matching of the observations to different physical sources. This is more likely to occur in regions of high source surface density (e.g. in the Galactic plane) or for close binary systems (either real or due to perspective effects). To identify poor astrometric solutions in their solar neighbourhood catalogue, Gaia Collaboration (2021b) constructed a randomforest classifier that assigns a probability to each source of having a good (or bad) astrometric solution based on astrometric quantities and quality indicators. The classification probability is approximately zero for sources with poor astrometric solutions, and approximately one for sources with good astrometric solutions. Gaia Collaboration (2021b) applied this classifier to stars 


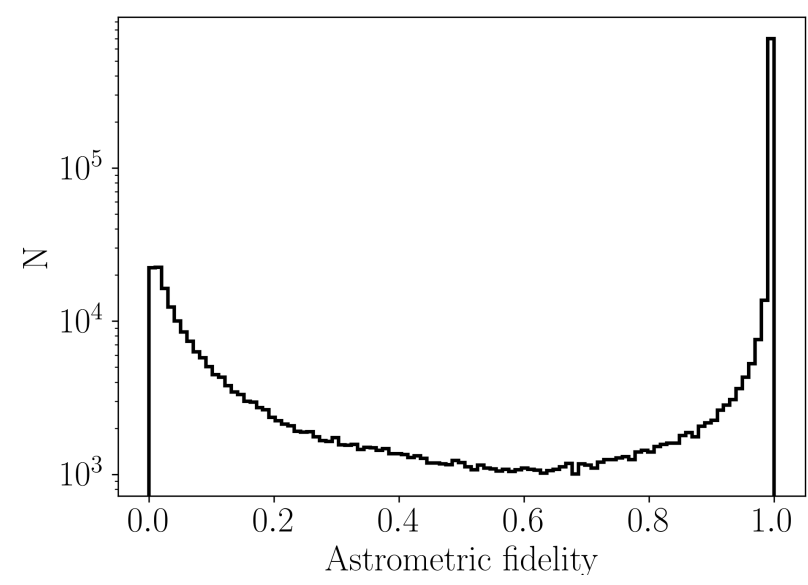

Fig. 10. Astrometric fidelity distribution for the sources in our target sample. Low astrometric fidelity values $(<0.5)$ correspond to bad astrometric solutions, and high astrometric fidelity values $(>0.5)$ correspond to good astrometric solutions.

with distances less than 100 pc. Rybizki et al. (2021) used similar methods to develop a neural network classifier, which they applied to the entire Gaia EDR3 catalogue. The distribution (in logarithmic scale) of 'astrometric fidelities' for the sources in our target sample is shown in Fig. 10. We select sources with astrometric fidelities $>0.5$ (around $75 \%$ of the target sample) following the definition of 'good' and 'bad' astrometric solutions in Rybizki et al. (2021). The distribution in the Galactic plane of sources with good and bad astrometric solutions is shown in Fig. 8 of Rybizki et al. (2021).

While this procedure allows us to obtain a more reliable sample, it might also eliminate binaries. Binaries however do not constitute the focus of this paper and do not affect the space distribution of our filtered sample.

\subsection{Kinematic cleaning}

Our astro-kinematic distance estimates are not accurate for sources that do not follow the disc kinematics assumed by our model. Such sources can be grouped into two categories:

1. Stars whose kinematics is influenced by the bar. These are located mostly towards the Galactic centre, and have astro-kinematic distances larger than Bailer-Jones photogeometric distances. These stars could in principle be young. Their astro-kinematic distance estimates could however be wrong due to the kinematic effects of the bar itself, which have not been included in our model (see Sect. 3 and Appendix B). In the following sections we focus on the in-plane distribution of stars within $d_{\text {kin }} \lesssim 5 \mathrm{kpc}$, and thus we do not consider sources in the bar/bulge regions.

2. Stars with heated vertical kinematics. These stars have Bailer-Jones photo-geometric distances $d_{\mathrm{BJ}} \gtrsim 3 \mathrm{kpc}$ and astro-kinematic distances $d_{\text {kin }} \sim 2 \mathrm{kpc}$. They could correspond to old contaminants, such as the RR Lyrae-type variable stars discussed in Sect. 2.2. To remove them we proceed as follows. First, we compute their vertical velocity, $v_{z}$ (see Appendix C). We fit the vertical velocity distribution with a Gaussian Mixture model with two components, the first (component A) with mean vertical velocity $\left\langle v_{z}\right\rangle_{A}=$ $-0.5 \mathrm{~km} \mathrm{~s}^{-1}$ and velocity dispersion $\sigma_{v_{z}, A}=7.0 \mathrm{~km} \mathrm{~s}^{-1}$ which has properties comparable with the young population that we are interested in studying, and the second (component B), with $\left\langle v_{z}\right\rangle_{B}=1.6 \mathrm{~km} \mathrm{~s}^{-1}$ and velocity dispersion $\sigma_{v_{z}, B}=29 . \mathrm{km} \mathrm{s}^{-1}$, which instead better reproduces the properties of an old population. We estimate the probability of each star belonging to either component A or B, and we select those stars with a greater probability of belonging to component A.

Figure 9 (right) shows the comparison between the astrokinematic distances and Bailer-Jones photo-geometric distances after cleaning the target sample as explained above. At this point, around $95 \%$ of the sources have astro-kinematic distances consistent with Bailer-Jones photo-geometric distances within $1 \sigma$.

Finally, using our astro-kinematic distances, we estimate the absolute magnitude of each star in the $K_{s}$ band, $M_{K_{s}}$, and we select stars with $M_{K_{s}}<0$ mag. This condition refines Eq. (1), and is designed to select stars of type earlier than B7V. After applying the quality cuts described in this section, our filtered sample reduces to 435273 stars.

\section{Three-dimensional space distribution}

To create the 3D density maps, we follow the method outlined in Zari et al. (2018). We compute galactic Cartesian coordinates, $x, y$, and $z$, for all the sources using our newly estimated astrokinematic distances and we define a box $V=12 \times 12 \times 1.5 \mathrm{kpc}$ centred on the Sun. We divide the cube into volume elements $v=5 \times 5 \times 5 \mathrm{pc}$. After computing the number of stars in each volume, we estimate the star density $D(x, y, z)$ by smoothing the distribution by means of a 3D Gaussian filter, using a technique similar to that used by Bouy \& Alves (2015). The Gaussian width (equal on the three axes) is $w=5 \mathrm{pc}$ and the Gaussian is truncated at $3 \sigma$. The choice of a certain $w$ value is arbitrary. A high $w$ value produces a smooth, less detailed map, while a low $w$ value results in a noisy map.

Figure 11 shows the projection on the Galactic plane of the filtered sample selected in Sect. 4. A number of features are visible, showing different structures at different scales and distances. At the centre of the map $(x, y \sim 0,0)$, a low-density gap is visible. This is due to a combination of reasons. First, in the solar neighbourhood the number of OBA stars is low. The Sun is indeed currently located in a cavity, partially filled with hot, lowdensity gas, which is usually called the Local Bubble (LB). The LB was likely created 10-20 Myr ago by a number of supernova explosions that likely halted any low- or high-mass star formation episode in the region. Second, our selection criteria exclude by construction stars of later spectral type than $\sim \mathrm{B} 7 \mathrm{~V}$. Thus, even if some late-type B stars are present within $200-300 \mathrm{pc}$ of the Sun, they are excluded from our selection. Third, there are selection effects due to the fact that (a) nearby bright sources are not included in Gaia EDR3 and that (b) some of those that are included might have poor 2MASS photometry; these latter are excluded by the colour selection in Eq. (2).

The small dense clumps can be associated to well-studied OB associations, for example Cygnus, Carina, Cassiopeia, and Vela are visible. The distribution of the lower density contours traces the Milky Way young disc structure, in particular the spiral arm location (see Sects. 5.2 and 6). The density distribution presents numerous low-density gaps. These could be due to the fact that our view might be obscured by interstellar dust towards certain lines of sight, however some gaps are located in regions of relatively low extinction (see also Sect. 5.3). For example, the Perseus gap $(x, y \sim-2,1 \mathrm{kpc})$ has been detected in the distribution of other young tracers, which are traditionally associated with spiral arms, such as the HII maps (Hou \& Han 2014) and the Cepheid distribution (Skowron et al. 2019, and see 


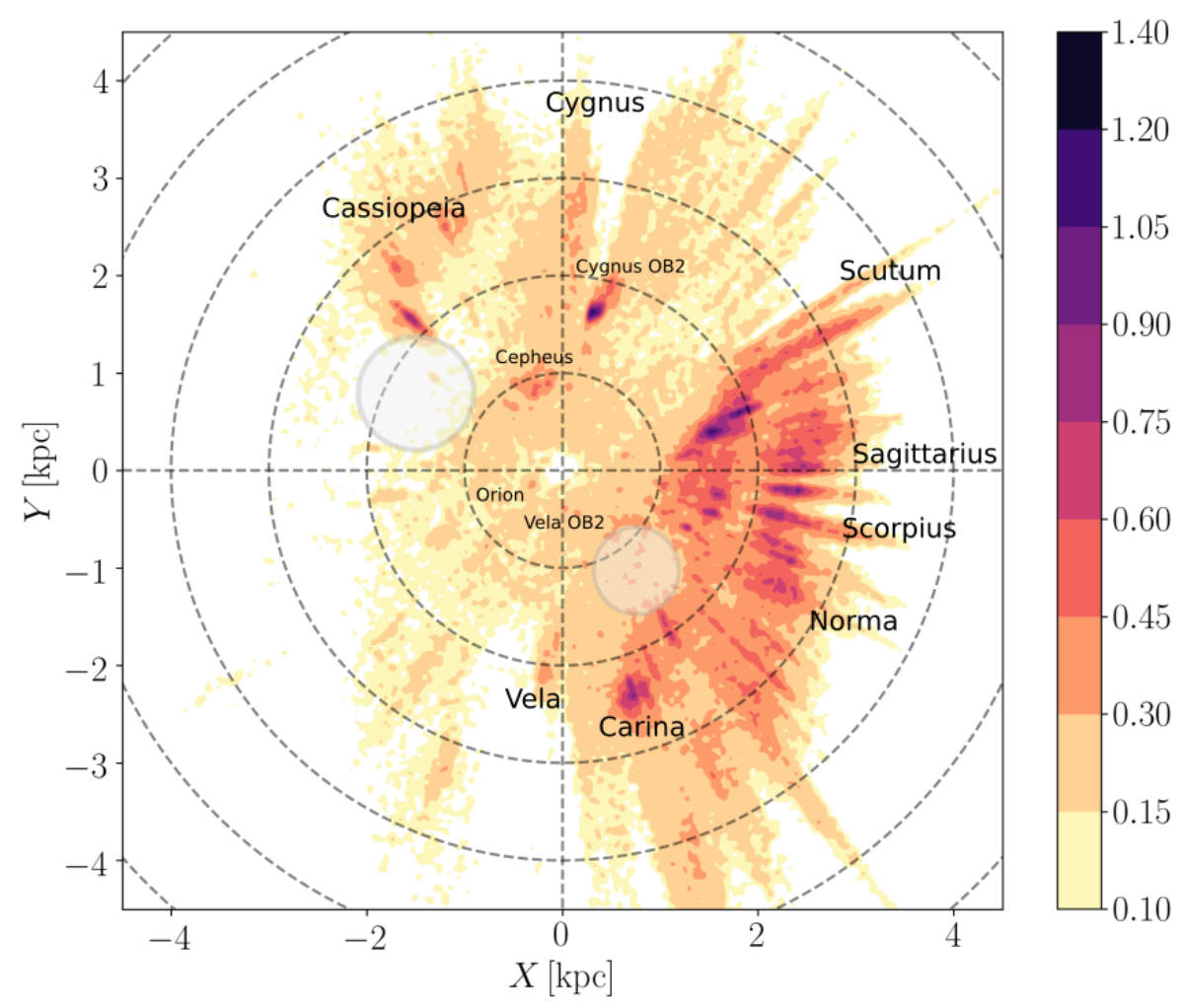

Fig. 11. Surface density of the stars selected in Sect. 3 projected on the Galactic plane. The Sun is in $(0,0)$, the $x$-axis is directed towards the Galactic centre, and the $y$-axis towards Galactic rotation. The $z$-axis is perpendicular to the plane. The density is displayed in arbitrary units. The dashed circles have radii from 1 to $6 \mathrm{kpc}$, in steps of $1 \mathrm{kpc}$. The labels follow the nomenclature proposed by K. Jardine (http:// gruze.org/galaxymap/map_2020/). The gaps in the density distribution mentioned in Sect. 5 are indicated by grey circles.
Sect. 5.4), and in the OB stars shown by Romero-Gómez et al. (2019) and Poggio et al. (2018, and see Sect. 5.2), and the highmass star-forming regions of Reid et al. (2014) and Reid et al. (2019, and see Sect. 5.5). By tracing the open cluster distribution in the Galactic disc, Cantat-Gaudin et al. (2020) suggest that a similar gap might be observed around $x, y \sim 1,-1 \mathrm{kpc}$, where our map shows an under-density, although less pronounced than the Perseus gap. In general, the distribution of stars along the 'arms' is not homogeneous, but characterised by under- and over-densities. For distances larger than $3-4 \mathrm{kpc}$, the number of sources (and thus their spatial density) decreases due to our magnitude limit at $G=16$ mag (see Sect. 2).

The radial features are caused by 'shadow cones' produced by foreground extinction, and by distance uncertainties. As shown in Fig. B.3, distance uncertainties are lower than $10 \%$ for stars within $5 \mathrm{kpc}$, and therefore are only partially responsible for the elongation.

\subsection{The Galactic warp}

As mentioned in Sect. 1, early-type star samples have been used to study the properties of the Galactic warp (see Poggio et al. 2018; Romero-Gómez et al. 2019, and references therein). Although in this paper we do not focus specifically on the warp, we studied the median height of our filtered sample with respect to the plane of the Galaxy and the median vertical velocity distribution, and compared our results to those by Poggio et al. (2018) and Romero-Gómez et al. (2019) to further validate our selection.

Figure 12 shows a map of the median height $z$ of our sample. The radial features are likely an artefact due to uneven sampling above or below the Galactic plane due to foreground extinction. Such features make the interpretation of Fig. 12 uncertain and may prevent us from seeing the warp shape, especially for $X>-2.5 \mathrm{kpc}$. For $X<-2.5 \mathrm{kpc}$, the impact of extinction is less dramatic (see also Fig. 14), and therefore the displacement visible above and below the plane might indeed trace the warp (see also Fig. 5 in Romero-Gómez et al. 2019).

The kinematic signature of the warp is evident in Fig. 13 (see e.g. Poggio et al. 2018; Romero-Gómez et al. 2019), which shows the median vertical velocity $\left(v_{z}\right)$ distribution of our filtered sample on the Galactic plane (see Appendix C for more details on how the vertical velocity was computed). For distances larger than $4 \mathrm{kpc}$ from the Sun, the vertical velocities have positive values that seem to peak at $Y \sim 0 \mathrm{kpc}$ and decrease towards both sides. At smaller distances from the Sun, some features also present positive vertical velocity. While some correspond to density enhancements in the 3D spatial distribution of our sources, this is not the case for all of them. Such variation in $v_{z}$ is likely related to the perturbations experienced by the disc (e.g. interaction with satellite galaxies).

\subsection{Comparison with other $O$ and $B$ star maps}

By combining Gaia EDR3 and VPHAS data with literature catalogues, Chen et al. (2019) obtained a sample of 14880 OB stars, earlier than B3V, which they used to describe the morphology of the spiral structure of the Milky Way. The method used by these latter authors to select $\mathrm{O}$ and $\mathrm{B}$ stars substantially differs from ours, but a qualitative comparison between their Fig. 4 and our maps shows substantially the same gross structures. This increases our confidence that both maps are revealing the same features in the Galactic O and B star distribution. The larger number of sources in our catalogue enables us to reveal more details in the source distribution. Interestingly, neither map shows a clear spiral structure. This is further discussed in the Sect. 6.

Figure 3 of Romero-Gómez et al. (2019) shows the distribution on the Galactic plane of their upper main sequence sources. Figure 11 of the present paper shows essentially the 


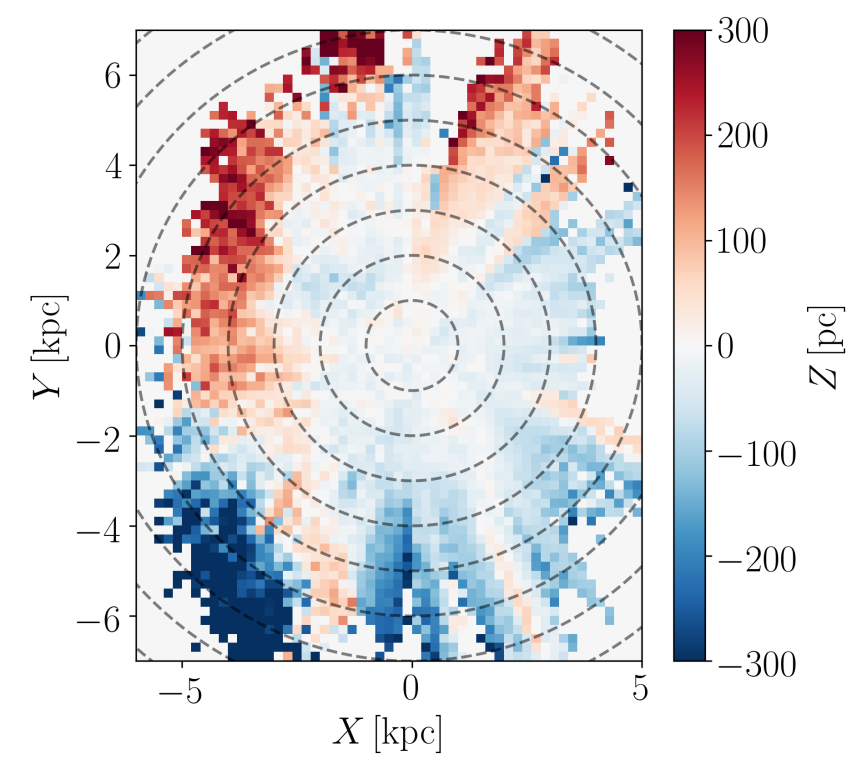

Fig. 12. Median height for the filtered sample projected on the Galactic plane. We divided the XY plane into bins of $200 \mathrm{pc}$ width, and we only show the ones containing more than ten stars. The dashed circles have radii from 1 to $9 \mathrm{kpc}$, in steps of $1 \mathrm{kpc}$.

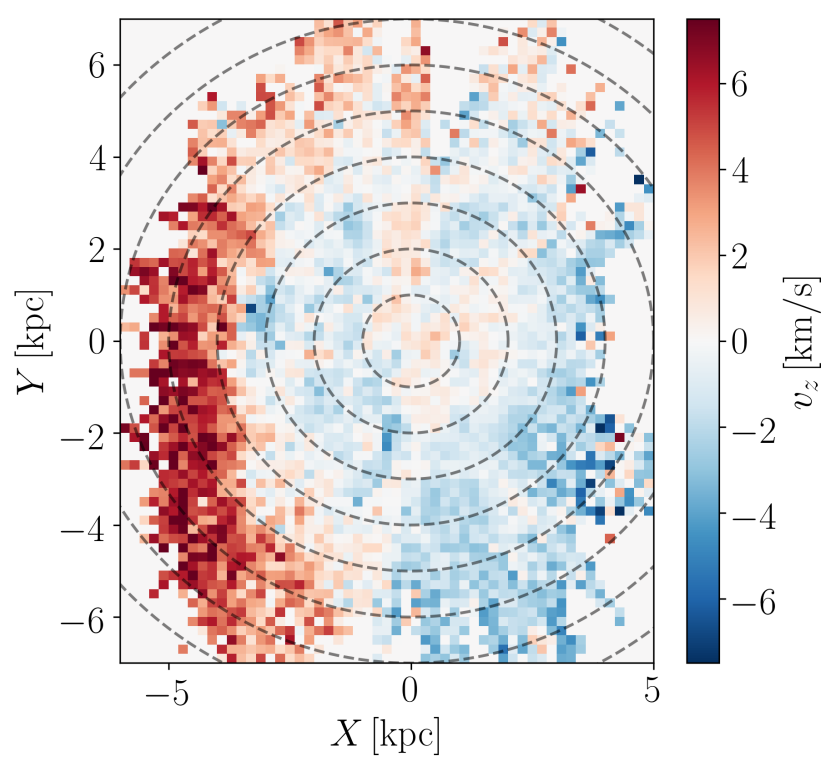

Fig. 13. Median vertical velocity $v_{z}$ distribution on the Galactic plane. We divided the XY plane into bins of $200 \mathrm{pc}$ width, and we only show the ones containing more than ten stars. The dashed circles have radii from 1 to $9 \mathrm{kpc}$, in steps of $1 \mathrm{kpc}$. The Galactic warp is visible for $R>$ $4 \mathrm{kpc}$ towards the Galactic anti-centre.

same over-densities, albeit in greater detail. This greater detail is mainly attributable to two factors: (a) to create their maps, Romero-Gómez et al. (2019) used a larger bin size than in the present work, and (b) their sample likely includes more later type stars than ours because of their selection criteria: this causes the distribution of sources to be smoother.

Figure 3 (panel A) in Poggio et al. (2018) also shows the distribution of upper main sequence sources on the Galactic plane. Similarly to Romero-Gómez et al. (2019), the pixel size of Poggio et al. (2018) is higher than ours. As mentioned above, this creates a smoother map, where it is not possible to identify small-scale over-densities. However, the comparison between large-scale structures shows many similarities.

\subsection{Comparison with dust distribution}

Dust is also a tracer of Galactic structure. Figure 14 shows the projection of the 3D dust distribution from Lallement et al. (2019) on the Galactic plane, over-plotted on top of our density map (see Fig. 11). The units of the density distribution are arbitrary. The dust density distribution shows discrepancies with respect to the star distribution. For example, the two elongated structures at the centre of the map are not prominent in the OB star distribution, and the dust features in the first and fourth Galactic quadrants are off-set with respect to the star density distribution, and seem to have a different separation and relative inclination. Such offsets are expected, as newly formed stars will drift from their birthplaces as they follow galactic rotation while the spiral arms move with a given pattern speed.

\subsection{Comparison with Cepheid distribution}

Classical Cepheids are young ( $<400 \mathrm{Myr})$ variable stars, whose distances can be estimated thanks to their period-luminosity relation. Figure 15 shows the Cepheids identified by Skowron et al. (2019) over-plotted on the density distribution of $\mathrm{O}$ and B stars (same as in Figs. 11 and 14). The colour-bar represents the ages (in Myr) determined by Skowron et al. (2019). The Cepheid distribution traces the density enhancements corresponding to the Sagittarius-Carina arm reasonably well (see Sect. 5.5), while the correspondence with the other density enhancements is not as tight. Although the age distribution of the Cepheids and our filtered sample is similar, the selection criteria are different: this makes a more direct comparison difficult.

\subsection{Comparison with maser distribution}

The spiral structure of the Milky Way can be traced also by water and methanol masers associated with high-mass-star-forming regions (HMSFRs). By measuring parallaxes and proper motions of such HMSFRs, Reid et al. (2019) provided estimates of the fundamental Galactic parameters, among which the pitch angle and the arm width. Figure 16 shows the masers and the location of the arms from the fit from Reid et al. (2019) on top of the OB star density map shown in Fig. 11. Similarly to the Cepheids distribution, there is good agreement between the position of the masers and the location of the most prominent over-densities, which makes us confident of our distance estimates. Unfortunately there are no available maser data tracing the spiral structure in the fourth Galactic quadrant $(X>0, Y<0)$, making a more detailed comparison in that region non-trivial. However, by tracing the spiral arms beyond the data while keeping the parameters fixed (dashed lines, and lighter contours), more discrepancies become visible, especially towards the inner Galaxy, towards the Sagittarius-Carina (purple) and Scutum-Centaurus arm (orange).

\section{Discussion}

We devised a large and systematically selected sample of massive, young stars and studied its properties focusing on the spatial distribution of our sources on the Galactic plane. In this section we discuss our findings in the context of the spiral arm structure of the Milky Way. 


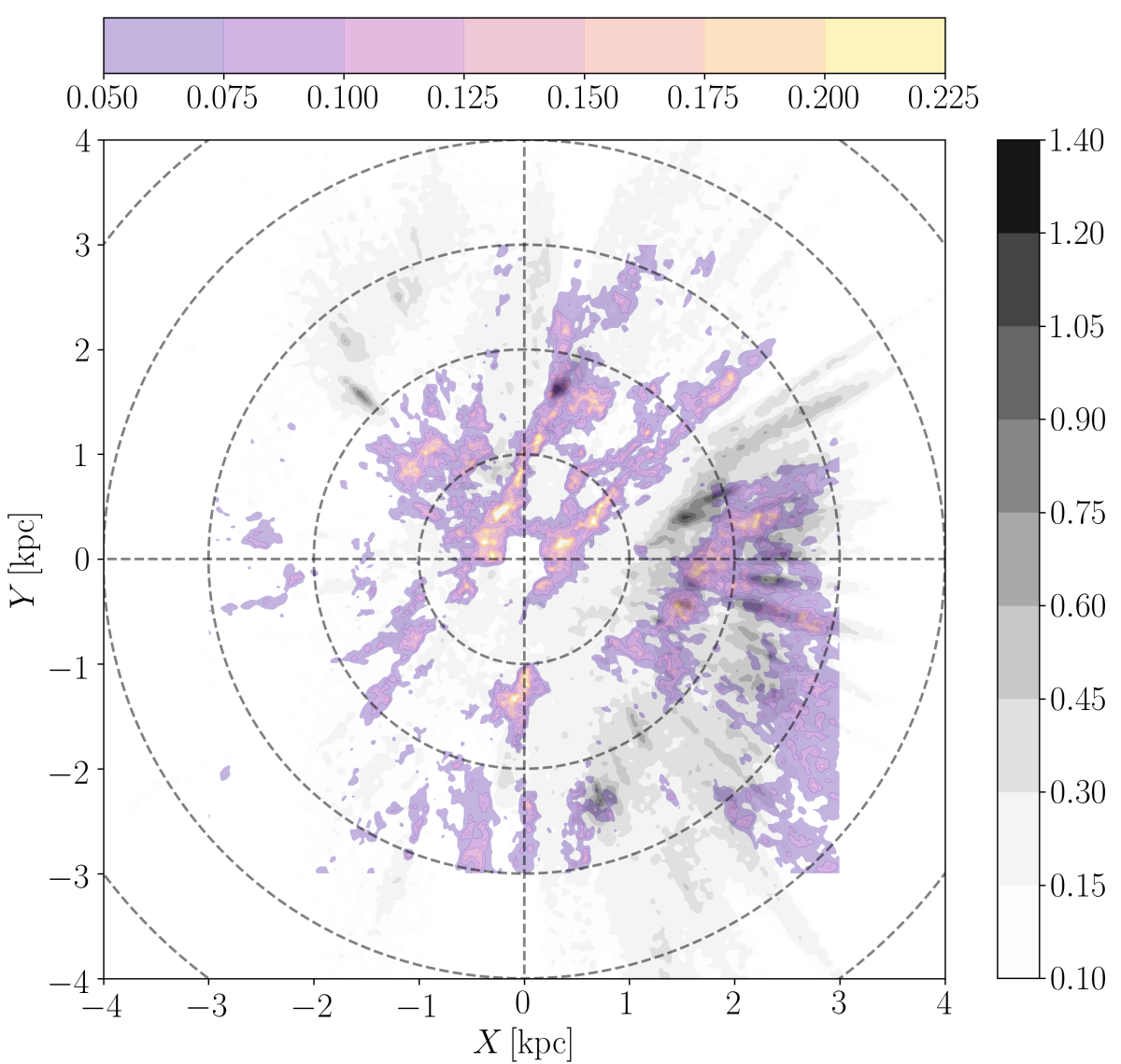

Fig. 14. Same as Fig. 11, with dust density contours from Lallement et al. (2019) overplotted. Both densities are displayed in arbitrary units. The dashed circles have radii from 1 to $5 \mathrm{kpc}$, in steps of $1 \mathrm{kpc}$.

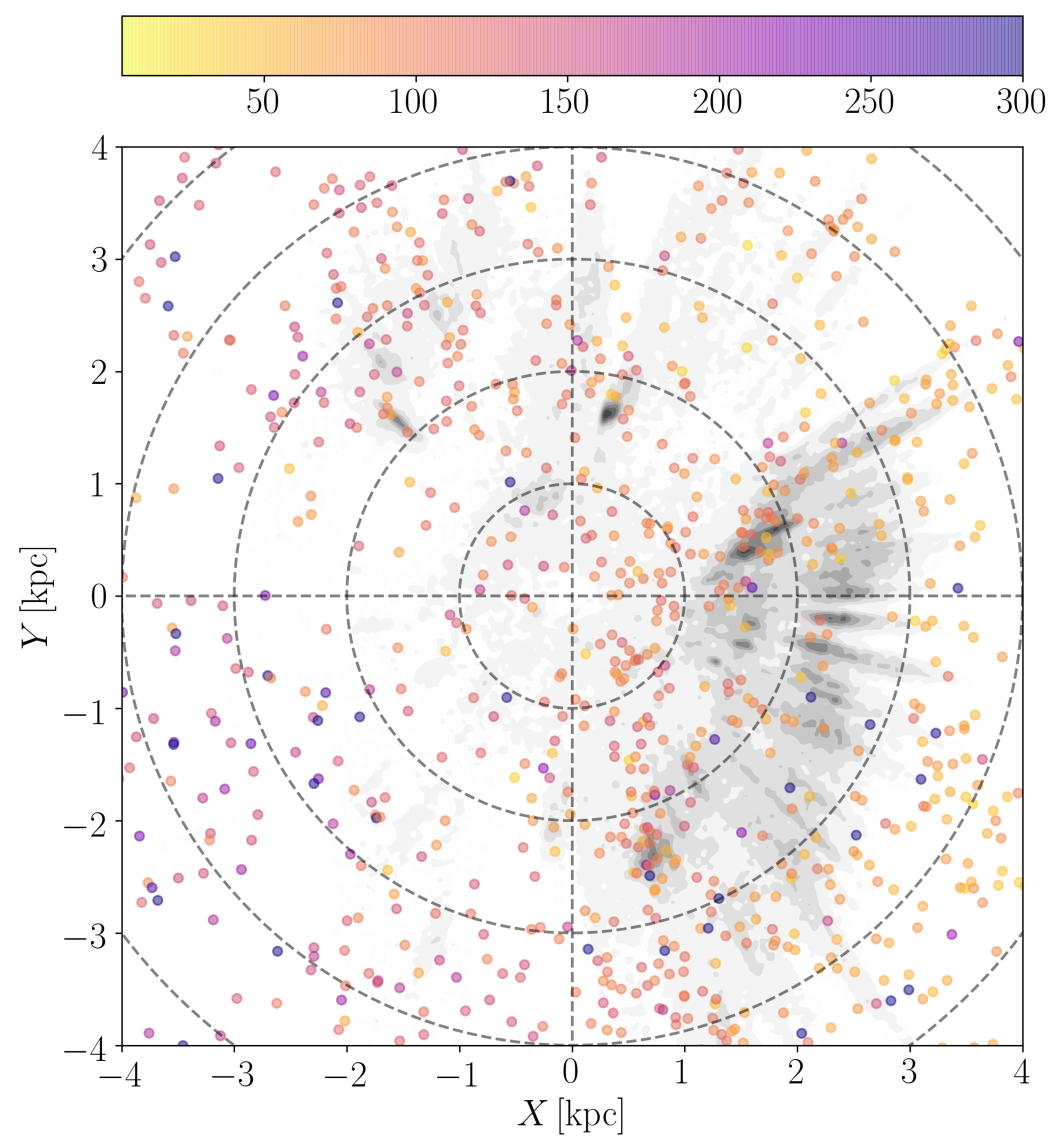

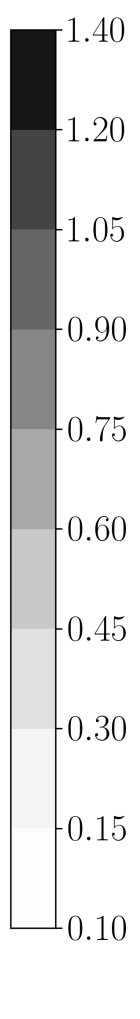

Fig. 15. Cepheids from Skowron et al. (2019), colour-coded by age (Myr). The density map is the same as Fig. 11. The dashed circles have radii from 1 to $6 \mathrm{kpc}$, in steps of $1 \mathrm{kpc}$. 


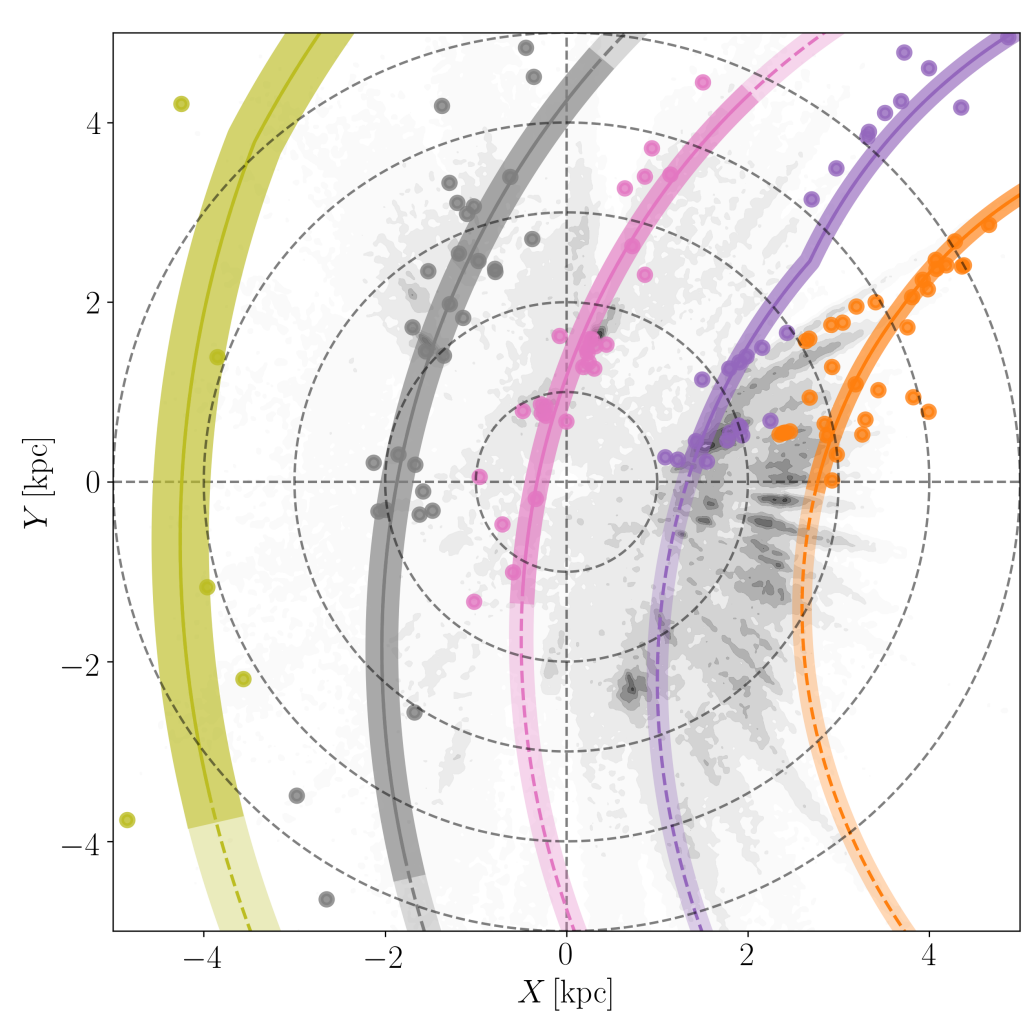

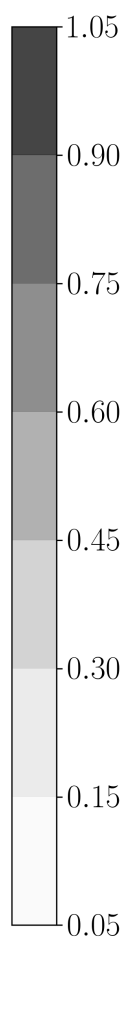

Fig. 16. Masers (dots) and the fit to the spiral arms (solid lines) from Reid et al. (2019) are over-plotted on top of our density map (same as Fig. 11). The maser distances were computed by naively inverting the parallax. The dashed circles have radii from 1 to $6 \mathrm{kpc}$, in steps of $1 \mathrm{kpc}$. The shaded regions correspond to the arm width. Different colours correspond to different spiral arms: Outer, green; Perseus, grey; Local, pink; Sagittarius-Carina, purple; ScutumCentaurus, orange.
Traditionally, the four main spiral arms of the Milky Way are considered to be Perseus, Sagittarius, Scutum, and Norma, with an additional Outer arm, which might be the outer part of one of the inner arms, and the Local or Orion arm, which is shorter and may be a spur or a bridge between two arms (see for instance Churchwell et al. 2009). In the Milky Way, the term 'spiral arm' has been used very broadly to refer to regions encompassing HI, molecular gas (and masers), dust, and young stars, while it has been shown from external galaxies that these tracers have quite distinct morphology. This has led to some inconsistencies in defining the spiral arms in our Galaxy. For example, the structure parameters derived by Reid et al. (2019) (see Fig. 16 and Sect. 5.5 of the present paper) do not agree with those presented in Chen et al. (2019) (see Sect. 5.2). Further, certain features might be interpreted as spiral arms or spurs connecting different arms: this is for example the case for the dust complex labelled 'Lower Sagittarius-Carina' by Lallement et al. (2019) (see Sects. 5.3 and Fig. 14) and 'Lower SagittariusCarina Spur' by Chen et al. (2020). Further, by studying the correlation between the location of young clusters and molecular clouds in NGC 7793 and M51 (respectively a flocculent and a grand-design spiral galaxy), Grasha et al. (2018, 2019) found that the star clusters that are associated (i.e. located within the footprint of a giant molecular cloud) are young, with a median age of 2 Myr in NGC 7793 and of 4 Myr in M51. Older clusters are mostly unassociated with any molecular cloud. Therefore, equating the same spiral arm morphology with different tracers (such as early type stars and dust) might be inappropriate.

The over-density towards the inner Galaxy (visible for positive $x$ values, i.e. in the first and fourth Galactic quadrants) in Fig. 11 is associated with the Sagittarius-Carina and ScutumCentaurus arm, and is much more prominent than the others, containing numerous HMSFRs. On the contrary, the distribution of massive stars associated with the Perseus arm peaks mainly in the second Galactic quadrant (towards Cassiopeia). This is con- sistent with the findings of Reid et al. (2019), and would point to the conclusion that the Perseus arm as traced by O- and B-type stars is not a dominant arm, and might be dispersing in the field. This suggests that a recent, large-scale episode of (massive) star formation occurred in Sagittarius-Carina and Scutum-Centaurus, while in the other arms (Perseus and Local) stars formed earlier, except in a few isolated massive associations, such as Cygnus and Cassiopeia. To confirm this, we selected stars brighter than $M_{K_{s}}=-1 \mathrm{mag}$ and $M_{K_{s}}=-2 \mathrm{mag}$ (corresponding respectively to the $K_{s}$ absolute magnitude of B2V-type and B1V-type stars), and we evaluated their density on the plane following the procedure described in Sect. 5. The density maps obtained with these samples are shown in Fig. 17, where also the same map of Fig. 11 is shown for clarity. The density contours in Fig. 17 (left) trace the same dense structures as in Fig. 11 (we note that the contour levels are different), while the low-density contours substantially disappear. This is even more evident in Fig. 17 (right). This is expected, as intrinsically brighter stars are on average younger and thus have had less time to disperse in the field.

Drimmel (2000) and Drimmel \& Spergel (2001) studied the spiral arm features of the Milky Way in the far-infrared and nearinfrared and concluded that the near-infrared features were consistent with a two-armed spiral model, while the other two arms were traced only by the far-infrared emission and thus could perhaps be present only in gas and young stars. More recently, Xu et al. (2018) and Chen et al. (2019) noted that the spiral arm structure traced by $\mathrm{O}$ - and early B-type stars may have many substructures in addition to the four major arms. These latter authors concluded that the Milky Way might not be a pure granddesign spiral galaxy with two or four well-defined, dominant arms. This might suggest that the Milky Way could exhibit characteristics of both flocculent and grand-design arms, with granddesign features seen in the infrared (old stellar populations) and more flocculent (or multi- arm) features seen in the optical (young stars). As mentioned above, these characteristics have 

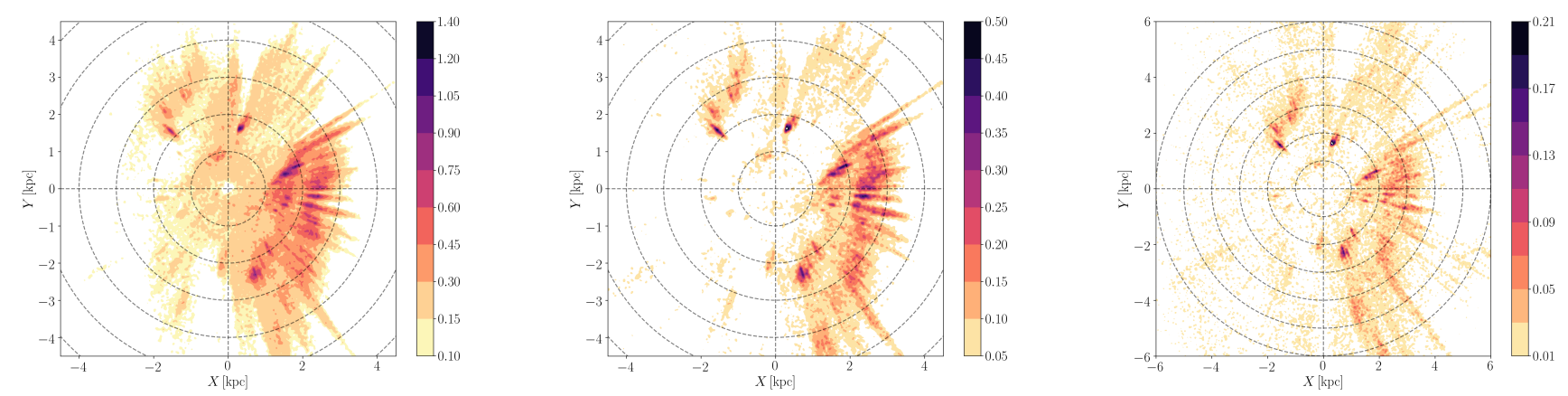

Fig. 17. Left: same as Fig. 11. Centre: density of stars with $M_{K_{s}}<-1$ mag projected on the Galactic plane. Right: density of stars with $M_{K_{s}}<-2$ mag projected on the Galactic plane. We note that the density scales are different.

been observed in spiral galaxies for instance by Kendall et al. (2011, 2015, and references therein), who concluded that galaxies that exhibit a grand-design structure in the optical also exhibit such structure in the near infra-red (NIR), while there are optically flocculent galaxies which also exhibit NIR grand-design structure. As pointed out by Dobbs \& Baba (2014), Elmegreen et al. $(1999,2011)$ noted nevertheless that most flocculent galaxies do not exhibit grand-design structure and those that do have very weak spiral arms. Elmegreen et al. (2003) further suggested that both grand-design and flocculent spirals (as seen in the old stars) exhibit a similar structure in the gas and young stars (independent of the underlying old stellar population) which is driven by turbulence in the disc. The co-existence of different spiral-arm patterns in the Milky Way disc might have different explanations. For example, Martos et al. (2004) concluded that secondary arms could be resonance-related features in the quasistationary density wave picture, while Steiman-Cameron et al. (2010) and Drimmel (2000) proposed that the traditional four spiral arms may represent the dynamical response of a gas disc to a two-arm spiral perturbation in the mass distribution.

As mentioned above, the density distribution presented in Fig. 11 exhibits a prominent density enhancement roughly corresponding to the Sagittarius-Carina and Scutum-Centaurus arms. This alone does not allow us to draw firm conclusions on the nature (or on the number) of the spiral arms of the Milky Way. On the one hand, star formation occurs in a clumpy and patchy fashion: this might explain the observed density distribution without necessarily assuming a flocculent structure. On the other hand, the map shown in Fig. 15 might suggest a different picture, in which the Milky Way, as traced by young stars ( $\$ 300 \mathrm{Myr})$, does not show a discrete set of spiral arms.

The spiral arm structure of the Milky Way can also be investigated using stellar kinematics (see e.g. Antoja et al. 2016). A spiral mass density perturbation can have a very different morphology from spiral-like density enhancements in molecular gas or young stars. Eilers et al. (2020) studied the kinematics of red giant stars and showed a spiral feature in their radial velocities that they interpret as arising from a perturbation to the potential of the Milky Way caused by a two-armed logarithmic spiral. Their model predicts the locations of the spiral perturbation in the Milky Way, with one roughly co-spatial to the Orion (or Local) arm, and the other roughly corresponding to the Outer arm. The location of the perturbation predicted by Eilers et al. (2020) does not closely follow the over-densities of the map in Fig. 11. The comparison between the radial velocities of the $\mathrm{O}$ - and B-type star sample and the giant sample will be crucial to make progress in our understanding of the mechanism giving rise to the spiral signature presented by Eilers et al. (2020).
The data of the SDSS-V survey will make such a comparison possible, and will also likely allow the construction of a surface density map of the giant sample which could be directly compared with the map presented in this work. The complete kinematic information that we will obtain by combining Gaia proper motions with SDSS-V radial velocities will also allow us to better separate stars belonging to different spiral arms, and to study their internal motions. Indeed, as already mentioned in Sect. 5, vertical velocities are not related to the perturbation in the disc kinematics induced by the spiral arms.

\section{Conclusions}

In this study we analyse the 3D space distribution of a sample of hot and luminous OBA stars, focusing in particular on their configuration in the Galactic disc. Our target selection is based on the combination of Gaia EDR3 astrometry and photometry and 2MASS photometry, and is designed to provide a well-defined selection function. We describe the properties of the target sample in terms of distribution in the sky, brightness, and variability. We estimate the purity and contamination of the catalogue by comparing with existing O- and B-type star catalogues, such as those by Liu et al. (2019), and Sota et al. (2014) and Maíz Apellániz et al. (2016), and by deriving stellar parameters $\left(T_{\text {eff }}\right.$ and $\log g$ ) for stars in common with LAMOST DR6.

By assuming that young massive stars are on near-circular orbits with a small velocity dispersion, we compute astrokinematic distances, and compare them to those derived by BailerJones et al. (2020). We use these distances to study the distribution of sources in the Galactic disc of a subset of the target sample, which we obtain by filtering out sources with spurious astrometric solutions and kinematic properties inconsistent with our model.

We find that the distribution of sources in the plane of the Milky Way is highly structured, characterised by over- and underdensities. Some of the density enhancements correspond to massive star forming regions, such as Carina, Cygnus, and Cassiopeia. With these associations, the inner arms (Sagittarius-Carina and Scutum-Centaurus) are strikingly more prominent in OBA stars than any of the outer arms, which we find little evidence for.

The distribution of O- and B-type stars from previous catalogues, classical Cepheids, dust, and HMSFRs shows similarities with our density distribution. However, the picture of the spiral arm structure of the Galaxy that we obtain in this study is complex and may suggest that young stars show little tendency to be neatly organised in distinct spiral features.

To assess different spiral arm models, it would be necessary to extend our map beyond the volume currently studied and, perhaps more importantly, to combine it with better Gaia data and spectroscopic information. 
The target sample that we have devised is indeed optimised for spectroscopic follow-up with the SDSS-V survey. The information that we will be able to obtain with SDSS-V will be crucial for studying the kinematics and dynamics of the spiral arms, and thus the nature of the spiral arms themselves. Finally, this sample will further enable the study of the properties of massive stars, for example in terms of multiplicity, internal structure, and (binary) evolution, in a way that is statistically robust.

Acknowledgements. We thank the referee for their comments, which improved the quality of this manuscript. We would also like to thank: J. Rybizki and $\mathrm{G}$. Green for making the catalogue of spurious sources available in advance of publication; A. Gould for discussions that prompted us to use kinematics for distance estimations; M. Sormani for discussions on the kinematics of the MW bar and bulge. This work has made use of data from the European Space Agency (ESA) mission Gaia (https://www.cosmos.esa.int/gaia), processed by the Gaia Data Processing and Analysis Consortium (DPAC, https://www. cosmos.esa.int/web/gaia/dpac/consortium). Funding for the DPAC has been provided by national institutions, in particular the institutions participating in the Gaia Multilateral Agreement. This publication makes use of data products from the Two Micron All Sky Survey, which is a joint project of the University of Massachusetts and the Infrared Processing and Analysis Center/California Institute of Technology, funded by the National Aeronautics and Space Administration and the National Science Foundation. Funding for the Sloan Digital Sky Survey V has been provided by the Alfred P. Sloan Foundation, the Heising-Simons Foundation, and the Participating Institutions. SDSS acknowledges support and resources from the Center for High-Performance Computing at the University of Utah. The SDSS web site is www.sdss.org. SDSS is managed by the Astrophysical Research Consortium for the Participating Institutions of the SDSS Collaboration, including the Carnegie Institution for Science, the Chilean Participation Group, the Gotham Participation Group, Harvard University, The Johns Hopkins University, L'Ecole polytechnique fédérale de Lausanne (EPFL), Leibniz-Institut für Astrophysik Potsdam (AIP), Max-PlanckInstitut für Astronomie (MPIA Heidelberg), Max-Planck-Institut für Extraterrestrische Physik (MPE), Nanjing University, National Astronomical Observatories of China (NAOC), New Mexico State University, The Ohio State University, Pennsylvania State University, Space Telescope Science Institute (STScI), the Stellar Astrophysics Participation Group, Universidad Nacional Autónoma de México, University of Arizona, University of Colorado Boulder, University of Illinois at Urbana-Champaign, University of Toronto, University of Utah, University of Virginia, and Yale University. The research leading to these results has (partially) received funding from the KU Leuven Research Council (gran C16/18/005: PARADISE), from the Research Foundation Flanders (FWO) under grant agreement G0H5416N (ERC Runner Up Project), as well as from the BELgian federal Science Policy Office (BELSPO) through PRODEX grant PLATO. E.P. acknowledges support from the Centre national d'études spatiales (CNES). This research made use of TOPCAT (Taylor 2005), Astropy, (Astropy Collaboration 2013, 2018), matplotlib (Hunter 2007), numpy (Harris et al. 2020), scipy (Virtanen et al. 2020), and scikit-learn (Pedregosa et al. 2011). This work would have not been possible without the countless hours put in by members of the open-source community all around the world.

\section{References}

Antoja, T., Roca-Fàbrega, S., de Bruijne, J., \& Prusti, T. 2016, A\&A, 589, A13 Astraatmadja, T. L., \& Bailer-Jones, C. A. L. 2016, ApJ, 832, 137 Astropy Collaboration (Robitaille, T. P., et al.) 2013, A\&A, 558, A33 Astropy Collaboration (Price-Whelan, A. M., et al.) 2018, AJ, 156, 123

Bailer-Jones, C. A. L., Rybizki, J., Fouesneau, M., Mantelet, G., \& Andrae, R. 2018, AJ, 156, 58

Bailer-Jones, C. A. L., Rybizki, J., Fouesneau, M., Demleitner, M., \& Andrae, R. 2020, AJ, 161, 147

Belokurov, V., Erkal, D., Deason, A. J., et al. 2017, MNRAS, 466, 4711

Bouwens, R. J., Illingworth, G. D., Oesch, P. A., et al. 2011, ApJ, 737, 90

Bouy, H., \& Alves, J. 2015, A\&A, 584, A26

Bowen, I. S., \& Vaughan, A. H. J. 1973, Appl. Opt., 12, 1430

Bressan, A., Marigo, P., Girardi, L., et al. 2012, MNRAS, 427, 127

Cantat-Gaudin, T., Anders, F., Castro-Ginard, A., et al. 2020, A\&A, 640, A1

Chen, B. Q., Huang, Y., Hou, L. G., et al. 2019, MNRAS, 487, 1400

Chen, B. Q., Li, G. X., Yuan, H. B., et al. 2020, MNRAS, 493, 351

Churchwell, E., Babler, B. L., Meade, M. R., et al. 2009, PASP, 121, 213

Deason, A. J., Belokurov, V., Erkal, D., Koposov, S. E., \& Mackey, D. 2017, MNRAS, 467, 2636

de Mink, S. E., Langer, N., Izzard, R. G., Sana, H., \& de Koter, A. 2013, ApJ, 764,166
Dobbs, C., \& Baba, J. 2014, PASA, 31, e035

Douglas, L. S., Bremer, M. N., Lehnert, M. D., Stanway, E. R., \& MilvangJensen, B. 2010, MNRAS, 409, 1155

Drimmel, R. 2000, A\&A, 358, L13

Drimmel, R., \& Spergel, D. N. 2001, ApJ, 556, 181

Drimmel, R., Smart, R. L., \& Lattanzi, M. G. 2000, A\&A, 354, 67

Eilers, A.-C., Hogg, D. W., Rix, H.-W., \& Ness, M. K. 2019, ApJ, 871, 120

Eilers, A. C., Hogg, D. W., Rix, H. W., et al. 2020, ApJ, 900, 186

Elmegreen, D. M., Chromey, F. R., Bissell, B. A., \& Corrado, K. 1999, AJ, 118, 2618

Elmegreen, B. G., Elmegreen, D. M., \& Leitner, S. N. 2003, ApJ, 590, 271

Elmegreen, D. M., Elmegreen, B. G., Yau, A., et al. 2011, ApJ, 737, 32

Frankel, N., Rix, H.-W., Ting, Y.-S., Ness, M., \& Hogg, D. W. 2018, ApJ, 865, 96

Frankel, N., Sanders, J., Rix, H.-W., Ting, Y.-S., \& Ness, M. 2019, ApJ, 884, 99 Gaia Collaboration (Prusti, T., et al.) 2016, A\&A, 595, A1

Gaia Collaboration (Brown, A. G. A., et al.) 2021a, A\&A, 649, A1

Gaia Collaboration (Smart, R. L., et al.) 2021b, A\&A, 649, A6

Grasha, K., Calzetti, D., Bittle, L., et al. 2018, MNRAS, 481, 1016

Grasha, K., Calzetti, D., Adamo, A., et al. 2019, MNRAS, 483, 4707

Gravity Collaboration (Abuter, R., et al.) 2018, A\&A, 615, L15

Gunn, J. E., Siegmund, W. A., Mannery, E. J., et al. 2006, AJ, 131, 2332

Haiman, Z., \& Loeb, A. 1997, ApJ, 483, 21

Harris, C. R., Millman, K. J., van der Walt, S. J., et al. 2020, Nature, 585, 357

Hopkins, P. F., Kereš, D., Oñorbe, J., et al. 2014, MNRAS, 445, 581

Hou, L. G., \& Han, J. L. 2014, A\&A, 569, A125

Hunter, J. D. 2007, Comput. Sci. Eng., 9, 90

Iorio, G., Belokurov, V., Erkal, D., et al. 2018, MNRAS, 474, 2142

Kendall, S., Kennicutt, R. C., \& Clarke, C. 2011, MNRAS, 414, 538

Kendall, S., Clarke, C., \& Kennicutt, R. C. 2015, MNRAS, 446, 4155

Kobulnicky, H. A., Kiminki, D. C., Lundquist, M. J., et al. 2014, ApJS, 213 , 34

Kollmeier, J. A., Zasowski, G., Rix, H. W., et al. 2017, ArXiv e-prints [arXiv:1711.03234]

Lallement, R., Babusiaux, C., Vergely, J. L., et al. 2019, A\&A, 625, A135

Langer, N., Schürmann, C., Stoll, K., et al. 2020, A\&A, 638, A39

Lindegren, L., Klioner, S. A., Hernández, J., et al. 2021a, A\&A, 649, A2

Lindegren, L., Bastian, U., Biermann, M., et al. 2021b, A\&A, 649, A4

Liu, Z., Cui, W., Liu, C., et al. 2019, ApJS, 241, 32

Mac Low, M.-M., \& Klessen, R. S. 2004, Rev. Mod. Phys., 76, 125

Maíz Apellániz, J., Sota, A., Arias, J. I., et al. 2016, ApJS, 224, 4

Martos, M., Hernandez, X., Yáñez, M., Moreno, E., \& Pichardo, B. 2004, MNRAS, 350, L47

Moe, M., \& Di Stefano, R. 2017, ApJS, 230, 15

O'Shaughnessy, R., Kim, C., Kalogera, V., \& Belczynski, K. 2008, ApJ, 672, 479

Pecaut, M. J., \& Mamajek, E. E. 2013, ApJS, 208, 9

Pedregosa, F., Varoquaux, G., Gramfort, A., et al. 2011, J. Mach Learn. Res., 12, 2825

Podsiadlowski, P., Joss, P. C., \& Hsu, J. J. L. 1992, ApJ, 391, 246

Poggio, E., Drimmel, R., Lattanzi, M. G., et al. 2018, MNRAS, 481, L21

Poggio, E., Drimmel, R., Andrae, R., et al. 2020, Nat. Astron., 4, 590

Reid, M. J., Menten, K. M., Brunthaler, A., et al. 2014, ApJ, 783, 130

Reid, M. J., Menten, K. M., Brunthaler, A., et al. 2019, ApJ, 885, 131

Riello, M., De Angeli, F., Evans, D. W., et al. 2021, A\&A, 649, A3

Robin, A. C., Reylé, C., Derriére, S., \& Picaud, S. 2003, A\&A, 409, 523

Romero-Gómez, M., Mateu, C., Aguilar, L., Figueras, F., \& Castro-Ginard, A. 2019, A\&A, 627, A150

Rybizki, J., Green, G., Rix, H. W., et al. 2021, ArXiv e-prints [arXiv:2101.11641]

Sana, H., de Mink, S. E., de Koter, A., et al. 2012, Science, 337, 444

Sana, H., Le Bouquin, J. B., Lacour, S., et al. 2014, ApJS, 215, 15

Schönrich, R., Binney, J., \& Dehnen, W. 2010, MNRAS, 403, 1829

Skowron, D. M., Skowron, J., Mróz, P., et al. 2019, Science, 365, 478

Skrutskie, M. F., Cutri, R. M., Stiening, R., et al. 2006, AJ, 131, 1163

Smee, S. A., Gunn, J. E., Uomoto, A., et al. 2013, AJ, 146, 32

Sota, A., Maíz Apellániz, J., Morrell, N. I., et al. 2014, ApJS, 211, 10

Steiman-Cameron, T. Y., Wolfire, M., \& Hollenbach, D. 2010, ApJ, 722, 1460

Tang, J., Bressan, A., Rosenfield, P., et al. 2014, MNRAS, 445, 4287

Taylor, M. B. 2005, in Astronomical Data Analysis Software and Systems XIV, eds. P. Shopbell, M. Britton, \& R. Ebert, ASP Conf. Ser., 347, 29

Van Bever, J., \& Vanbeveren, D. 2000, A\&A, 358, 462

Virtanen, P., Gommers, R., Oliphant, T. E., et al. 2020, Nat. Meth., 17, 261

Wilson, J. C., Hearty, F. R., Skrutskie, M. F., et al. 2019, PASP, 131, 055001

Xu, Y., Hou, L.-G., \& Wu, Y.-W. 2018, Res. Astron. Astrophys., 18, 146

Zari, E., Hashemi, H., Brown, A. G. A., Jardine, K., \& de Zeeuw, P. T. 2018, A\&A, 620, A172 


\section{Appendix A: Query of the Gaia archive}

Here we provide an example query for cross-matching 2MASS and Gaia EDR3 in the Gaia archive using the cross-match with Gaia DR2.

select edr3.* xdr2.*, tm.*

from gaiaedr3.gaia_source as edr3

inner join gaiaedr3.dr2_neighbourhood as $\mathrm{xdr} 2$

on edr3.source_id = xdr2.dr3_source_id

inner join gaiadr2.tmass_best_neighbour as xtm

on xdr2.dr2_source_id = xtm. source_id

inner join gaiadr1.tmass_original_valid AS tm

on tm.tmass_oid = xtm.tmass_oid

WHERE xtm.angular_distance $<1$.

AND xdr2.angular_distance $<100$.

AND edr3.phot_g_mean_mag $<16$.

AND edr3.parallax $<\operatorname{power}\left(10,\left(10-\mathrm{tm} . \mathrm{ks} \_\mathrm{m}\right) / 5\right)$

\section{Appendix B: Astro-kinematic distances}

In this section we describe all the terms of Eq. (8), which we report here for convenience:

$p\left(d \mid \boldsymbol{o}, m_{K_{s}} \Theta_{\mathrm{KM}}, \Theta_{\mathrm{SM}}, \Theta_{\mathrm{CMD}}\right) \propto$

$p\left(\boldsymbol{o} \mid \Theta_{K M}\right) p\left(d, m_{K_{s}} \mid l, b, \Theta_{\mathrm{SM}}, \Theta_{\mathrm{CMD}}\right)$.

The prior $p\left(d, m_{K_{s}} \mid l, b, \Theta_{\mathrm{SM}}, \Theta_{\mathrm{CMD}}\right)$ can be written as:

$p\left(d, m_{K_{s}} \mid l, b, \Theta_{\mathrm{SM}}, \Theta_{\mathrm{CMD}}\right) \propto p\left(d \mid l, b, \Theta_{\mathrm{SM}}\right) f\left(d, m_{K_{s}} \mid \Theta_{\mathrm{CMD}}\right)$.

The term $p\left(d \mid l, b, \Theta_{\mathrm{SM}}\right)$ represents the probability density function of observing a star in the direction $(l, b)$ at distance $d$ from the Sun according to our assumed model for the spatial distribution of stars in the Galaxy. The term $f\left(d, m_{K_{s}} \mid \Theta_{\mathrm{CMD}}\right)$ specifies the fraction of stars that can be observed at a distance $d$ and magnitude $m_{K_{s}}$ given the distribution of stars in colour-magnitude space and our selection criteria. In the following sections, we describe the different components of Eq. (B.1).

Figure B.1 shows example $p d f$ s for four randomly selected stars. Both the parallaxes of the stars in the top row have $\sigma_{\varpi} / \varpi<$ $20 \%$. In the left panel, the distance estimate is mostly constrained by the term $P\left(d \mid \mu_{l^{*},} \Theta_{\mathrm{KM}}\right)$, which represent the probability of observing a star at distance $d$ given the observed proper motion along Galactic latitude and the kinematic model $\Theta_{\mathrm{KM}}$. $P\left(d \mid \mu_{l^{*}}, \Theta_{\mathrm{KM}}\right)$ shows bi-modality, with a primary maximum at $\sim 6 \mathrm{kpc}$, and a secondary maximum at $\sim 1.8 \mathrm{kpc}$. The total distance $p d f$ shows traces of such bi-modality. In the right panel of the top row on the other hand, the parallax component dominates the distance determination. The star in the left panel of the bottom row has $\sigma_{\varpi} / \varpi<20 \%$, however both $P\left(d \mid \mu_{l^{*}}, \Theta_{\mathrm{KM}}\right.$ and $P\left(d \mid \mu_{b}, \Theta_{\mathrm{KM}}\right.$ peak at closer distances than the parallax term, and thus put a strong constraint on the final distance estimate. Finally, the star in the right panel of the bottom row has $\sigma_{\varpi} / \varpi \approx 70 \%$. The final distance $p d f$ is nevertheless still quite narrow, as a result of the combination of the different terms.

\section{B.1. Kinematic model}

We assume that stars in our sample follow the rotation curve determined by Eilers et al. (2019),

$v_{c}=229 \mathrm{~km} \mathrm{~s}^{-1}-1.7 \mathrm{~km} \mathrm{~s}^{-1} \mathrm{kpc}^{-1}\left(R-R_{\odot}\right)$,

with $R$ the Galactocentric radius and $R_{\odot}=8.122 \mathrm{kpc}$ (Gravity Collaboration 2018). The intrinsic velocity dispersions at the
Sun's location in the components of the velocity in Galactic coordinates $U, V, W$ are $\sigma_{U}=16.7 \mathrm{~km} \mathrm{~s}^{-1}, \sigma_{V}=10.8 \mathrm{~km} \mathrm{~s}^{-1}$, and $\sigma_{W}=6 \mathrm{~km} \mathrm{~s}^{-1}$ (Robin et al. 2003). These values are assumed to be constant over the disc.

In Cartesian Galactic coordinates, the $p d f$ of the space velocity for one star in our sample is:

$p\left(\boldsymbol{v} \mid \Theta_{\mathrm{KM}}\right)=\frac{1}{(2 \pi)^{3 / 2}|S|^{1 / 2}} \exp \left(-\frac{1}{2}(\boldsymbol{v}-\boldsymbol{u})^{\prime} S^{-1}(\boldsymbol{v}-\boldsymbol{u})\right)$,

the prime signifying the transpose of the vector. The quantity $\boldsymbol{u}$ takes into account Galactic rotation and solar motion, and can be written as:

$\boldsymbol{u}=\boldsymbol{v}_{c}\left(\boldsymbol{x}_{\mathrm{star}}\right)-\boldsymbol{v}_{c}\left(\boldsymbol{x}_{\odot}\right)-\boldsymbol{v}_{\odot}$,

where

$\boldsymbol{v}_{c}\left(\boldsymbol{x}_{\mathrm{star}}\right)=v_{c}\left(r_{\mathrm{star}}\right) \cdot\left(\hat{\boldsymbol{x}}_{\mathrm{star}} \times \boldsymbol{e}_{\mathrm{Z}}\right)$

is the circular velocity in Galactocentric Cartesian coordinates of a star at a Galactocentric distance $r_{\text {star }}$ and $\boldsymbol{x}_{\text {star }}=$ $\left[x_{\text {star }}, y_{\text {star }}, z_{\text {star }}\right]$. The unit vectors $\hat{\boldsymbol{x}}_{\text {star }}$ and $\boldsymbol{e}_{Z}$ are defined as $\hat{\boldsymbol{x}}_{\text {star }}=\boldsymbol{x}_{\text {star }} /\left|\boldsymbol{x}_{\text {star }}\right|$ and $\boldsymbol{e}_{Z}=[0,0,1]$. At the Sun's position $\boldsymbol{x}_{\odot}$,

$\boldsymbol{v}_{c}\left(\boldsymbol{x}_{\odot}\right)=v_{c}\left(r_{\odot}\right) \boldsymbol{e}_{Y}$

where $\boldsymbol{e}_{Y}=[0,1,0]$ is the unit vector in the $Y$ direction in Galactic coordinates. We assumed the Sun's position with respect to the Galactic centre to be $x_{\odot}=[-8.122,0 ., 0.025] \mathrm{kpc}$ and the Sun's peculiar motion with respect to the Local Standard of Rest to be $\boldsymbol{v}_{\odot}=[11.1,12.24,7.25] \mathrm{km} \mathrm{s}^{-1}$ (Schönrich et al. 2010). We write the velocity dispersion matrix $S$ as:

$$
\left(\begin{array}{ccc}
\sigma_{U}^{2} & 0 & 0 \\
0 & \sigma_{V}^{2} & 0 \\
0 & 0 & \sigma_{W}^{2}
\end{array}\right)
$$

For each star, the astrometric observables are the parallax $\varpi$, and the proper motions components in $l$ and $b, \mu_{l *}=\mu_{l} \cos b$, and $\mu_{b}$ respectively. These are collected in arrays:

$\boldsymbol{o}=\left(\begin{array}{c}\varpi-\varpi_{0} \\ \mu_{l *} \\ \mu_{b}\end{array}\right)$

where $\varpi_{0}$ is the systematic zero point offset of Gaia EDR3 parallaxes. There is not a unique $\varpi_{0}$ for all the Gaia EDR3 stars. Following Bailer-Jones et al. (2020), we applied the parallax zeropoint correction derived by Lindegren et al. (2021b).

By assuming Gaussian errors, the $p d f$ for the observables $\boldsymbol{o}$ given the true values $\tilde{\boldsymbol{o}}$, is then:

$p(\boldsymbol{o} \mid \tilde{\boldsymbol{o}})=\frac{1}{(2 \pi)^{3 / 2}|C|^{1 / 2}} \exp \left(-\frac{1}{2}(\boldsymbol{o}-\tilde{\boldsymbol{o}})^{\prime} C^{-1}(\boldsymbol{o}-\tilde{\boldsymbol{o}})\right)$.

The true values are defined as:

$\tilde{\boldsymbol{o}}=\left(\begin{array}{c}1 / d \\ \boldsymbol{p}^{\prime} \cdot \boldsymbol{v} / d A \\ \boldsymbol{q}^{\prime} \cdot \boldsymbol{v} / d A\end{array}\right)$

where $d$ is the true distance to the star and $A=$ $4.74047 \mathrm{~km} \mathrm{yr} \mathrm{s}^{-1}$. The two vectors $\boldsymbol{p}$ and $\boldsymbol{q}$ are the two components of the normal triad in longitude and latitude, and, as above, 

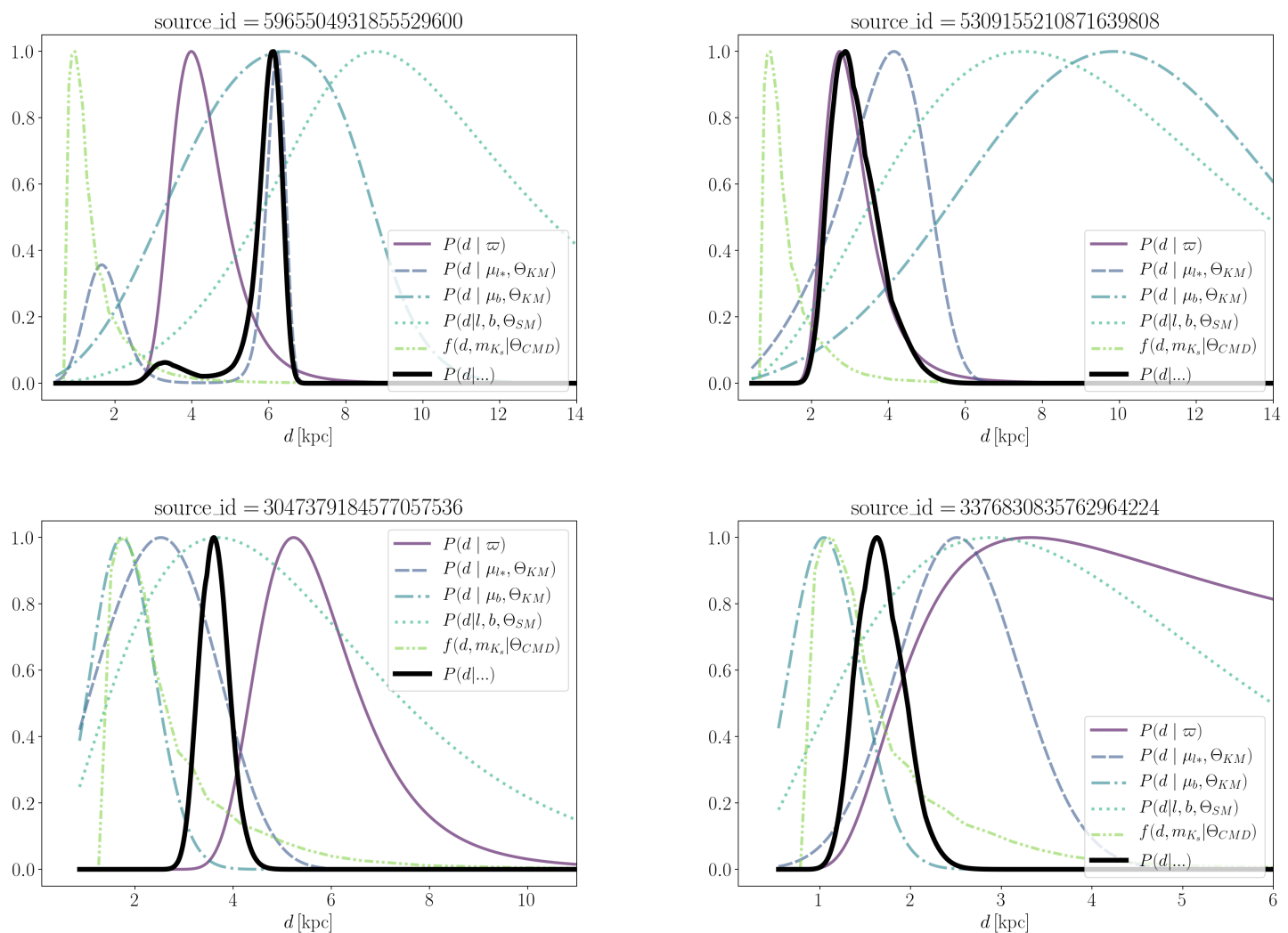

Fig. B.1. Posterior $p d f \mathrm{~s}$ for four stars in our sample (their Gaia EDR3 source_id is displayed at top of each panel). The thick black solid line represents $p\left(d \mid \boldsymbol{o}, m_{K_{s}} \Theta_{\mathrm{KM}}, \Theta_{\mathrm{SM}}, \Theta_{\mathrm{CMD}}\right)$. The coloured thin lines illustrate the different components of the $p d f$ s. The correlation terms in the covariance matrix of Eq. (B.12) are neglected here for illustrative purposes. The distributions are scaled so that they peak at unity.

the prime signify their transpose. The elements of the astrometric covariance matrix $C$ are:

$\left(\begin{array}{ccc}\sigma_{\varpi}^{2} & \rho_{\varpi \mu_{l *}} \sigma_{\varpi} \sigma_{\mu_{l} *} & \rho_{\varpi \mu_{b}} \sigma_{\varpi} \sigma_{\mu_{b}} \\ \rho_{\varpi \mu_{l *}} \sigma_{\varpi} \sigma_{\mu_{l} *} & \sigma_{\mu_{l} *}^{2} & \rho_{\mu_{l *} \mu_{b}} \sigma_{\mu_{l} *} \sigma_{\mu_{b}} \\ \rho_{\varpi \mu_{b}} \sigma_{\varpi} \sigma_{\mu_{b}} & \rho_{\mu_{l} * \mu_{b}} \sigma_{\mu_{l} *} \sigma_{\mu_{b}} & \sigma_{\mu_{b}}^{2}\end{array}\right)$,

and are provided in the Gaia archive.

The joint $p d f$ of the observables with the velocity is therefore:

$p(\boldsymbol{o}, \boldsymbol{v} \mid d)=p(\boldsymbol{o} \mid \tilde{\boldsymbol{o}}(\boldsymbol{v}, d)) p\left(\boldsymbol{v} \mid \Theta_{\mathrm{KM}}\right)$.

The $p d f$ for the observables is obtained by marginalising over the velocity:

$p\left(\boldsymbol{o} \mid \Theta_{\mathrm{KM}}\right)=\int_{\infty}^{+\infty} d^{3} \boldsymbol{v} p(\boldsymbol{o} \mid \tilde{\boldsymbol{o}}(\boldsymbol{v}, d)) p\left(\boldsymbol{v} \mid \Theta_{\mathrm{KM}}\right)$.

The integral can be resolved analytically. Since the product of two normal $p d f$ is normal and the marginal density of a normal $p d f$ is also normal, we can write:

$p\left(\boldsymbol{o} \mid \Theta_{K M}\right)=\frac{1}{(2 \pi)^{3 / 2}|D|^{1 / 2}} \exp \left(-\frac{1}{2}(\boldsymbol{o}-\boldsymbol{c})^{\prime} D^{-1}(\boldsymbol{o}-\boldsymbol{c})\right)$,

where

$\boldsymbol{c}=\left(\begin{array}{c}1 / d \\ \boldsymbol{p}^{\prime} \cdot \boldsymbol{u} / d A \\ \boldsymbol{q}^{\prime} \cdot \boldsymbol{u} / d A\end{array}\right)$,

and

$D=C+\left(\begin{array}{ccc}0 & 0 & 0 \\ 0 & \boldsymbol{p}^{\prime} S \boldsymbol{p} & \boldsymbol{p}^{\prime} S \boldsymbol{q} \\ 0 & \boldsymbol{q}^{\prime} S \boldsymbol{p} & \boldsymbol{q}^{\prime} S \boldsymbol{q}\end{array}\right)$

\section{B.2. Structural model}

The probability of a star to be at a true distance $d$ is proportional to the stellar density $\rho$ predicted by our spatial model $\Theta_{\mathrm{SM}}$, so that we can write:

$p\left(d \mid l, b, \Theta_{\mathrm{SM}}\right) \propto d^{2} \rho\left(d \mid l, b, \Theta_{\mathrm{SM}}\right)$,

where the Jacobian term $d^{2}$ takes volume effects into account. For convenience we write our model in Galactocentric coordinates $(R, \phi, z)$, where the stellar density is modelled as an exponential disc:

$\rho(R, \phi, z)=\rho_{0} \exp \left(-\frac{R-R_{\odot}}{L}\right) \times \exp \left(-\frac{|z|}{h_{z}}\right)$.

Here, $R$ and $z$ are the Galactocentric radius and height above the plane. The quantity $h_{z}=0.15 \mathrm{kpc}$ is the disc scale height (cf. Poggio et al. 2020), and $L=3.5 \mathrm{kpc}$ is the best fit scalelength of the young $(<1 \mathrm{Gyr})$ disc, where we have used the model presented in Frankel et al. (2018) with the data and method in Frankel et al. (2019).

\section{B.3. Luminosity function}

We assume that the Milky Way has a universal luminosity function $\phi\left(M_{K_{s}}\right)=\phi\left(M_{K_{s}}\left(m_{K_{s}}, d\right)\right)$, where $M_{K_{s}}$ is the absolute magnitude in the $2 \mathrm{MASS} K_{s}$ band, and $M_{K_{s}}=m_{K_{s}}-5 \log _{10}(d)+5$. Contrary to Poggio et al. (2020) or Astraatmadja \& Bailer-Jones (2016), we use the near-infrared $K_{s}$ band to be able to neglect extinction, at least as a first approximation. Following the procedure outlined by Poggio et al. (2020), we construct the distribution of stars in colour-magnitude space $\phi\left(m_{K_{s}}, d, c\right)$ by assuming 


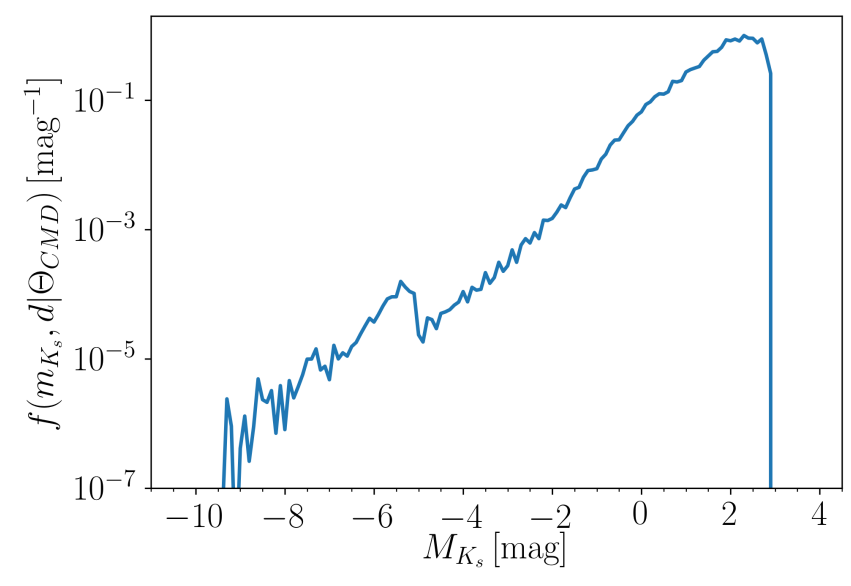

Fig. B.2. Effective luminosity function $f\left(d, m_{K_{s}} \mid \Theta_{\mathrm{CMD}}\right)$ used to determine our prior, normalised to unity.

a constant star formation rate, the two-part power law Kroupa initial mass function corrected for unresolved binaries, and solar metallicity. The term $c$ indicates a colour (for instance, $G-K_{s}$ ). The distribution of stars in colour-magnitude space is then modified by setting to zero the parts of the distribution where $T_{\text {eff }}<8000 \mathrm{~K}$. This roughly corresponds to applying the colour cuts aiming at selecting hot stars as described in Sect. 2.1, and effectively changes the term $S(c)$ of Eq. (B.20) into a selection approximately based on effective temperature, $S\left(T_{\text {eff }}\right)$. We can thus write the effective luminosity function, shown in Fig. B.2, $f\left(d, m_{K_{s}} \mid \Theta_{\mathrm{CMD}}\right)$ as:

$f\left(d, m_{K_{s}} \mid \Theta_{\mathrm{CMD}}\right)=\int \phi\left(m_{K_{s}}, d, c\right) S(c) d c$,

where $S(c)=1$ if $T_{\text {eff }}>8000 \mathrm{~K}$ and $S(c)=0$ otherwise.

\section{B.4. Distance uncertainties}

Figure B.3 shows the fractional distance uncertainty for stars in the filtered sample (see Sect. 4). For distances smaller than $\sim 4 \mathrm{kpc}$, parallax uncertainties dominate the fractional error distribution. This is a regime where $\sigma_{\varpi} / \varpi<0.1$, and the inverse parallax is a good distance estimate (see also Bailer-Jones et al. 2020). For distances larger than $\sim 4 \mathrm{kpc}$, the kinematic model and the other priors described above keep the distance uncertainties from exploding. The fractional errors are below the $10 \%$ for $d_{\text {kin }}<5 \mathrm{kpc}$, and remain well below the $20 \%$ for $d_{\text {kin }}<10 \mathrm{kpc}$.

\section{B.5. Distance validation}

To validate our distances, we identified the stars in our sample that belong to the three clusters IC 4756, NGC 2112, and King 21 , respectively at $469 \mathrm{pc}, 1090 \mathrm{pc}$, and $3005 \mathrm{pc}$, as determined by Cantat-Gaudin et al. (2020). Figure B.4 shows the distance $p d f$ s of such stars. As expected, the $p d f$ s for stars at larger distances are broader than for those at closer distances. The distance estimates for all the cluster members are compatible within $2 \sigma$ with the average distance value. The dispersion around the median distance is between 10 and 20 pc for IC 4756, around $65 \mathrm{pc}$ for NGC 2112, and around $300 \mathrm{pc}$ for King 21. This indicates that distance uncertainties are within the $10 \%$ for $d \sim 3 \mathrm{kpc}$ (where most of the over-densities are located), in agreement with Appendix B.4.

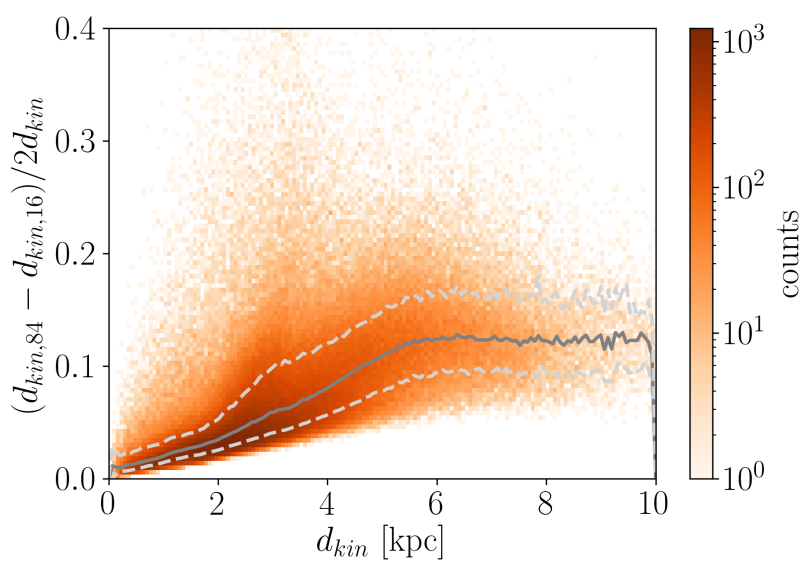

Fig. B.3. Fractional distance uncertainty $d_{\text {kin, } 84}-d_{\text {kin, } 16} / 2 d_{\text {kin }}$ for stars in the clean sample. The dashed light grey lines correspond to the 16 th and 84th percentiles, the solid grey line corresponds to the median fractional error.

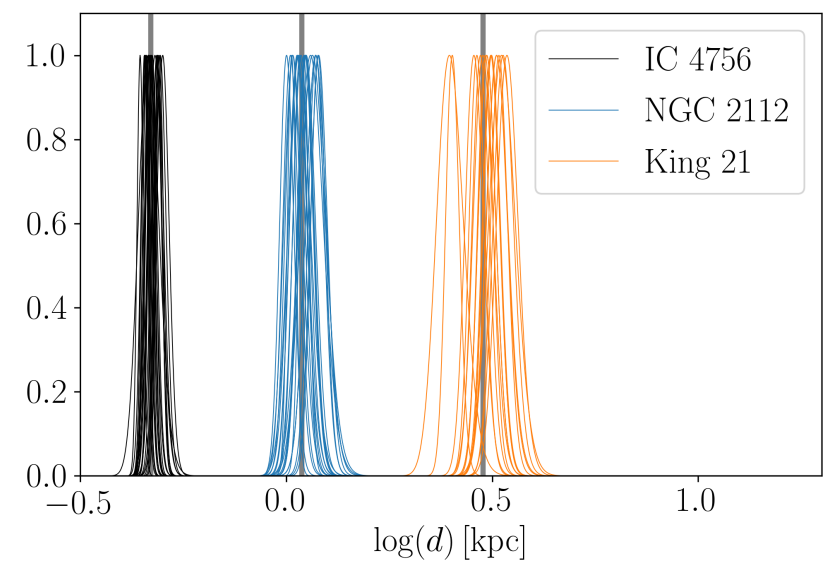

Fig. B.4. Single star distance $p d f$ s for the members of the three clusters IC 4756 (black), NGC 2112 (blue), and King 21 (orange). The grey vertical lines represent the cluster distances (respectively $469 \mathrm{pc}, 1090 \mathrm{pc}$, and 3005 pc) derived by Cantat-Gaudin et al. (2020).

\section{Appendix C: Vertical velocity}

As described in Drimmel et al. (2000), the vertical velocity $v_{z}$ of a star is computed by:

$v_{z}=4.74047 \mu_{b} d / \cos (b)+W_{\odot}+v_{r} \sin (b)$,

where $b$ is Galactic longitude, $\mu_{b}$ is the proper motion along $b$, $d$ is the distance estimate, $W_{\odot}$ is the Sun's velocity along $Z$, and $v_{r}$ is the line-of-sight velocity. The majority of stars in our sample lack line-of-sight velocities, and therefore it is not possible to directly calculate their vertical velocity. However, for stars at low Galactic latitudes $\left(|b|<20^{\circ}\right)$, the term $v_{r} \sin (b)$ can be approximately computed by assigning to each star the velocity it would have if it followed the Galactic rotation curve exactly:

$v_{r} \sin (b) \approx\left(S-S_{\odot}\right) \tan (b)$,

where: $S_{\odot}=U_{\odot} \cos l+V_{\odot} \sin (l)$, with $l$ the galactic longitude of a star, and $U_{\odot}$ and $V_{\odot}$ the components of the Sun's motion along $X$ and $Y$ respectively; $S=v_{\phi} R_{\odot} / R-v_{\mathrm{LSR}} \sin (l)$, with $R_{\odot}$ the distance of the Sun from the Galactic Centre, $R$ a star's Galactocentric radius, $v_{\phi}$ the azymutal velocity, and $v_{\mathrm{LSR}}$ the standard of rest velocity. We assumed $(U, V, W)_{\odot}=(11.1,12.24,7.25) \mathrm{km} \mathrm{s}^{-1}$ from Schönrich et al. (2010), and the rotation curve derived by Eilers et al. (2019), reported in Eq. (B.3). 